\title{
ON BALLISTIC DIFFUSIONS IN RANDOM ENVIRONMENT
}

\author{
Lian SHEN \\ Department of Mathematics, ETH-Zurich, CH-8092 Zurich, Switzerland
}

Received 14 May 2002, accepted 13 February 2003

ABSTRACT. - In this article we investigate diffusions in random environment. We provide a sufficient condition for a strong law of large numbers with non-vanishing limiting velocity and a functional central limit theorem. In the course of this work we introduce certain regeneration times and obtain a renewal structure. As an illustration, we apply our results to a class of anisotropic gradient-type diffusions in random environment, where the technique of the environment viewed from the particle does not apply well.

○ 2003 Éditions scientifiques et médicales Elsevier SAS

RÉSUMÉ. - Cet article traite des diffusions en milieu aléatoire. On donne une condition suffisante pour la loi forte des grands nombres avec une vitesse limite non nulle et pour un théorème limite central fonctionnel. Certains temps de régénération sont introduits et une sructure de renouvellement est obtenue. A titre d'illustration, nous appliquons nos techniques à une classe de diffusions anisotropes de type gradient pour lesquelles la technique de l'environnement vu de la particule ne s'applique pas bien.

( 2003 Éditions scientifiques et médicales Elsevier SAS

\section{Introduction}

Random motions in random media has been a very active research area over the last twenty years, both in the discrete and continuous settings. The method of the "environment viewed from the particle" has played an important role, see for instance $[13,16,19,21,23]$. In the continuous setting, there has been a special emphasis on the gradient-type or the incompressible drift situations, and most of the progress has occurred when there is an explicit invariant measure for the process of the environment viewed from the particle, which is absolutely continuous with respect to the static distribution of the random medium, see [5,15,17,20,21,23], see however [14]. Nevertheless, the general setting is still poorly understood. On the other hand, progress has been made recently in the discrete setting, see [3,4,33-36]. One appeal of the continuous theory is that, unlike in the discrete setting (cf. [3]), imposing independence assumptions on the environment at the level of bonds or sites, is not relevant anymore. 
Related to this feature, some arguments of the discrete theory are not applicable to the continuous setting.

The present article investigates diffusions in random environment in the continuous setting, in situations where a priori no invariant measure of the process of the environment viewed from the particle is known to exist. We provide a sufficient condition, under which the process satisfies a strong law of large numbers with nonvanishing velocity, which can further be refined by a central limit theorem. In particular, under this condition, the diffusion in random environment exhibits a ballistic behavior. We use a strategy which has been successful in the discrete setting. We construct certain regeneration times which provide a renewal structure, see [35]. As an application of our results, we show the ballistic behavior of a concrete class of diffusion processes in random environment, which is a natural generalization of some discrete models mentioned in [18], which were studied in [27].

We now describe the setting in more details. We denote with $(\Omega, \mathscr{A}, \mathbb{P})$ a probability space and with $G=\left\{t_{x}: x \in \mathbb{R}^{d}\right\}$ a group of measure preserving transformations, acting ergodically on $\Omega$, for details see the beginning of Section 2 . We consider bounded measurable functions $b(\cdot): \Omega \rightarrow \mathbb{R}^{d}$ and $\sigma(\cdot): \Omega \rightarrow \mathbb{R}^{d \times d}$, as well as two constants $\bar{b}, \bar{\sigma}>0$ such that

$$
|b(\omega)| \leqslant \bar{b}<\infty, \quad|\sigma(\omega)| \leqslant \bar{\sigma}<\infty,
$$

where $|\cdot|$ denotes Euclidean norm both for vectors and $d \times d$-matrices. We write

$$
b(x, \omega)=b\left(t_{x}(\omega)\right), \quad \sigma(x, \omega)=\sigma\left(t_{x}(\omega)\right) .
$$

We assume that $b(\cdot, \omega)$ and $\sigma(\cdot, \omega)$ are Lipschitz continuous, i.e., there exists a constant $K>0$ such that for all $\omega \in \Omega, x, y \in \mathbb{R}^{d}$,

$$
\begin{aligned}
& |b(x, \omega)-b(y, \omega)| \leqslant K|x-y| \quad \text { and } \\
& |\sigma(x, \omega)-\sigma(y, \omega)| \leqslant K|x-y| .
\end{aligned}
$$

Further, we assume that $\sigma \sigma^{t}(x, \omega)$ is uniformly elliptic, that means, there is a constant $v>0$ such that for all $x, y \in \mathbb{R}^{d}$ and $\omega \in \Omega$,

$$
\frac{1}{v}|y|^{2} \leqslant\left|\sigma^{t}(x, \omega) y\right|^{2} \leqslant v|y|^{2},
$$

where $\sigma^{t}$ stands for the transposed matrix of $\sigma$. For a Borel subset $F \subset \mathbb{R}^{d}$, we define the $\sigma$-algebra generated by $b(x, \omega), \sigma(x, \omega)$, for $x \in F$ :

$$
\mathscr{H}_{F} \stackrel{\text { def }}{=} \sigma\{b(x, \omega), \sigma(x, \omega): x \in F\},
$$

and assume an independence condition, which we call $R$-separation. Namely, there exists an $R>0$, such that for all Borel subsets $F, F^{\prime}$ in $\mathbb{R}^{d}$ with

$$
d\left(F, F^{\prime}\right) \stackrel{\text { def }}{=} \inf \left\{\left|x-x^{\prime}\right|: x \in F, x^{\prime} \in F^{\prime}\right\}>R,
$$




$$
\mathscr{H}_{F} \text { and } \mathscr{H}_{F^{\prime}} \text { are } \mathbb{P} \text {-independent. }
$$

Let us mention two examples of such random vectors $b(x, \omega)$ and random matrices $\sigma(x, \omega)$ respectively. The convolution of a Poissonian point process with a Lipschitz continuous vector-valued, or matrix-valued, function supported in a ball of radius $R / 2$ yields after truncation a possible example, cf. [31], p. 185. Another possible example is to use the Gaussian field, described in [1], Sections 1.6 and 2.3. After convolution and truncation, we get another example. (The formula (2.3.4) on p. 28 in [1] need be changed to $X(x)=\int g(x-\lambda) d Z(\lambda)$, where $g(\lambda)$ is some vector- or matrix-valued Lipschitz continuous function, compactly supported in a ball of radius $R / 2$.)

We denote by $\left(C\left(\mathbb{R}_{+}, \mathbb{R}^{d}\right), \mathscr{F}, \mathrm{W}\right)$ the canonical Wiener space, and with $\left(W_{t}\right)_{t \geqslant 0}$ the canonical Brownian motion (which is independent from $(\Omega, \mathscr{A}, \mathbb{P})$ ). The diffusion process in the random environment $\omega$ is the law $\mathrm{P}_{x}^{\omega}$ (which is sometimes called the quenched law) on $\left(C\left(\mathbb{R}_{+}, \mathbb{R}^{d}\right), \mathscr{F}\right)$ of the solution of the stochastic differential equation:

$$
\left\{\begin{array}{l}
d X_{t}(\omega)=b\left(X_{t}, \omega\right) d t+\sigma\left(X_{t}, \omega\right) d W_{t} \\
X_{0}=x, \quad x \in \mathbb{R}^{d}, \omega \in \Omega
\end{array}\right.
$$

The aim of this article is to study the asymptotic properties of $X$. under the "annealed law":

$$
\mathrm{P}_{x} \stackrel{\text { def }}{=} \mathbb{P} \times \mathrm{P}_{x}^{\omega}
$$

We provide a sufficient condition, see (3.1-i), under which the strong law of large numbers holds, that is:

$$
\mathrm{P}_{0} \text {-a.s. } \quad \frac{X_{t}}{t} \rightarrow v, \quad \text { as } t \rightarrow \infty,
$$

where $v$ is a deterministic and non-vanishing velocity (cf. Theorem 3.2). Further, we show that the stronger condition (3.1-ii) guarantees a functional central limit theorem, namely as $s$ tends to infinity, the $C\left(\mathbb{R}_{+}, \mathbb{R}^{d}\right)$-valued process

$$
B_{.}^{s} \stackrel{\text { def }}{=} \frac{1}{\sqrt{s}}\left(X_{s .}-s v \cdot\right)
$$

converges in law, under the annealed measure $\mathrm{P}_{0}$, to a non-degenerate $d$-dimensional Brownian motion with covariance matrix $\mathbf{K}$ (cf. Theorem 3.3).

The derivation of this sufficient condition (3.1) is based on the strategy of constructing some regeneration times $\tau_{k}, k \geqslant 1$, similar to those defined in [35], and providing a renewal structure, cf. Theorem 2.5. The sufficient condition is then expressed in terms of the transience of the diffusion $X$. in some direction $\ell$ and the finiteness of the first (or the second) moment of $\tau_{1}$ conditioned on no-backtracking, cf. (3.1). There are several ways to construct these regeneration times $\tau_{k}$. In the spirit of $[4,36]$, we introduce additional Bernoulli variables. In essence, the first regeneration time $\tau_{1}$ is the first integer time, at which the diffusion process reaches a local maximum in a given direction $\ell \in S^{d-1}$, the auxiliary Bernoulli variable takes value 1 , and from then on the process never backtracks. The regeneration times $\tau_{k}, k \geqslant 2$, are then obtained by iteration of this procedure. For the true definition, we refer to (2.12)-(2.17), (2.22). In our construction we take special 
advantage of the diffusion structure to couple the Bernoulli variables with the diffusion process, the resulting renewal structure, cf. Theorem 2.5, gives us a good control over the trajectory of the diffusion, see Remark 2.6, and we also have a convenient Markov structure, cf. Corollary 2.2. This provides a key tool for studying asymptotic behavior of the diffusion in a random environment. Further applications of this renewal structure and Theorems 3.2, 3.3 will follow.

As an illustration of our results, we study a class of reversible diffusion processes, for which $\sigma=\mathbb{1}$ and $b(x, \omega)=\nabla V(x, \omega)$, where $V(\cdot, \omega)$ has uniformly bounded and Lipschitz continuous derivatives, and there exist a unit vector $\ell \in \mathbb{R}^{d}, A, B>0$ and $\lambda>0$ such that

$$
A \mathrm{e}^{2 \lambda \ell \cdot x} \leqslant \mathrm{e}^{2 V(x, \omega)} \leqslant B \mathrm{e}^{2 \lambda \ell \cdot x}, \quad \text { for all } x \in \mathbb{R}^{d} \text { and } \omega \in \Omega .
$$

In the case where $\lambda=0$, the diffusive behavior of the process has been extensively investigated, cf. [5,21,22], however we do not know of any result when $\lambda>0$. We show in this article that when $\lambda>0$, (no matter how small $\lambda$ is) the sufficient condition (3.1) is fulfilled (in fact, we prove the much stronger exponential estimates under $\widehat{\mathrm{P}}_{x}^{\omega}$, cf. Theorem 4.9 and Corollary 4.10, which can also be used to deduce certain large deviation controls, cf. [32,33]). As a result, the above mentioned law of large numbers and functional central limit theorem hold, see Theorem 4.11. The class under consideration includes the case where $b(x, \omega)=\nabla \widetilde{V}(x, \omega)+\lambda \ell$, for some bounded $\widetilde{V} \in C^{1}\left(\mathbb{R}^{d}, \mathbb{R}\right)$, with bounded and Lipschitz continuous derivatives. Let us mention that this situation is closely related to some of the models studied by Lebowitz and Rost in [18], where the existence of an effective limiting velocity is mentioned as an open question.

Let us also point out that Theorems 3.2, 3.3 have a scope which goes beyond the above class of examples. In particular in the discussion of the above examples, we obtain uniform controls in $\omega$ and we do not even need to take advantage of the fact that the moment conditions (3.1-i), (3.1-ii) in Theorems 3.2, 3.3 are expressed in terms of annealed measures (i.e., integrating over $\omega$ ). Further applications of Theorems 3.2, 3.3 will appear elsewhere.

Let us finally describe how this article is organized. In Section 2, we enlarge the probability space with coupled Bernoulli random variables, cf. Theorem 2.1. We then define the regeneration times $\left(\tau_{k}\right)_{k \geqslant 1}$, cf. (2.12)-(2.17), and we provide the crucial renewal structure in Theorem 2.5.

In Section 3, the sufficient condition is expressed in terms of the transience of the diffusion in the direction $\ell$ and the (square) integrability of $\tau_{1}$ conditioned on nobacktracking, cf. (3.1). With the help of the renewal structure constructed in Section 2, we are able to show the ballistic behavior of $\left(X_{t}\right)_{t \geqslant 0}$ in Theorem 3.2, and a functional central limit theorem in Theorem 3.3.

In Section 4, we will apply the results from the previous sections to the specific class of models described in (1.9). An important role is played by estimates on the exit distribution and exit time of the diffusion processes from a large cylinder with axis parallel to $\ell$, cf. Propositions 4.2 and 4.3. The main integrability properties of $X_{\tau_{1}}$ and $\tau_{1}$ are derived in Theorem 4.9 and Corollary 4.10, and our main result is stated in Theorem 4.11. 
Finally, in the appendix, we collect some results about continuous martingales and linear parabolic partial differential equations of second order, which are used throughout this article.

\section{The renewal structure}

In this section we will enlarge the probability space $\left(C\left(\mathbb{R}_{+}, \mathbb{R}^{d}\right), \mathscr{F}, \mathrm{P}_{x}^{\omega}\right)$ to $\left(C\left(\mathbb{R}_{+}\right.\right.$, $\left.\left.\mathbb{R}^{d}\right) \times\{0,1\}^{\mathbb{N}}, \mathscr{F} \otimes \mathscr{S}, \widehat{\mathrm{P}}_{x}^{\omega}\right)$, by adding some suitable auxiliary i.i.d. Bernoulli random variables, see (2.6) and Theorem 2.1.

On the enlarged space $\left(\Omega \times C\left(\mathbb{R}_{+}, \mathbb{R}^{d}\right) \times\{0,1\}^{\mathbb{N}}, \mathscr{A} \otimes \mathscr{F} \otimes \mathscr{S}, \widehat{\mathrm{P}}_{x}\right)$, see (2.11), we will define the regeneration times $\tau_{k}, k \geqslant 1$, and discover the resulting renewal structure under the new annealed measure $\widehat{P}_{0}$, see Theorems 2.4 and 2.5.

For the random environment $(\Omega, \mathscr{A}, \mathbb{P})$, we assume that for all $x, y \in \mathbb{R}^{d}, t_{x}$ is a mapping on $\Omega$ with $t_{0}=1$ and $t_{x+y}=t_{x} \circ t_{y}$; the mapping $(x, \omega) \mapsto t_{x}(\omega)$ is $(\mathscr{B} \otimes \mathscr{A}, \mathscr{A})$-measurable, with $\mathscr{B}$ denoting the Borel $\sigma$-algebra on $\mathbb{R}^{d} ; t_{x}$ preserves the $\mathbb{P}$-measure; and for $A \in \mathscr{A}$ such that $t_{x}(A)=A$ for all $x$, then $\mathbb{P}[A] \in\{0,1\}$. We recall that under these assumptions $\left\{t_{x}: x \in \mathbb{R}^{d}\right\}$ is a group of strongly continuous unitary operators on $L^{2}(\Omega, \mathscr{A}, \mathbb{P})$, cf. p. 223 in [12].

\subsection{The coupling construction}

We first need to introduce further notations. Let $\ell \in \mathbb{R}^{d}$ be a given unit vector, and let

$$
U^{x} \stackrel{\text { def }}{=} B_{6 R}(x+5 R \ell), \quad B^{x} \stackrel{\text { def }}{=} B_{R}(x+9 R \ell),
$$

be the two subsets shown in Fig. 1.

We also introduce for open set $G \subset \mathbb{R}^{d}, u \in \mathbb{R}$ the $\left(\mathscr{F}_{t}\right)_{t \geqslant 0}$-stopping times $\left(\left(\mathscr{F}_{t}\right)_{t \geqslant 0}\right.$ denotes the canonical right continuous filtration on $\left.\left(C\left(\mathbb{R}_{+}, \mathbb{R}^{d}\right), \mathscr{F}\right)\right)$ :

$$
\left\{\begin{array}{l}
T_{G} \stackrel{\text { def }}{=} \inf \left\{t \geqslant 0: X_{t} \notin G\right\}, \\
T_{u} \stackrel{\text { def }}{=} \inf \left\{t \geqslant 0: \ell \cdot\left(X_{t}-X_{0}\right) \geqslant u\right\}, \\
\widetilde{T}_{u} \stackrel{\text { def }}{=} \inf \left\{t \geqslant 0: \ell \cdot\left(X_{t}-X_{0}\right) \leqslant u\right\},
\end{array}\right.
$$

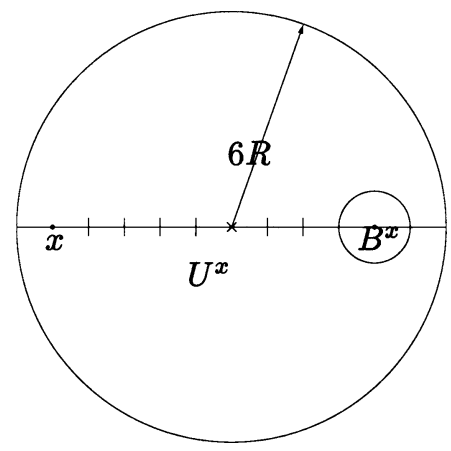

Fig. 1. Sets $U^{x}$ and $B^{x}$. 
and the maximal relative displacement to $X_{0}$ the process $\left(\ell \cdot X_{s}\right)_{s \geqslant 0}$ has reached within time $t$,

$$
M(t) \stackrel{\text { def }}{=} \sup \left\{\ell \cdot\left(X_{s}-X_{0}\right): 0 \leqslant s \leqslant t\right\} .
$$

We denote by $p_{\omega}(s, x, y)$ the transition density under $\mathrm{P}_{x}^{\omega}$, which is a continuous function of $s>0, x, y \in \mathbb{R}^{d}$ such that $\mathrm{P}_{x}^{\omega}\left[X_{s} \in G\right]=\int_{G} d y p_{\omega}(s, x, y)$, for all open set $G \subset \mathbb{R}^{d}$, cf. [8], pp. 139-141. We also introduce the sub-transition density $p_{\omega, U^{x}}(s, x, y)$, which is a continuous function in $s>0, x \in \mathbb{R}^{d}$ and $y \in U^{x}$, fulfilling:

$$
\mathrm{P}_{x}^{\omega}\left[X_{s} \in G, T_{U^{x}}>s\right]=\int_{G} d y p_{\omega, U^{x}}(s, x, y),
$$

for all open set $G \subset U^{x}$.

Under our assumptions on the drift term $b(\cdot, \omega)$ and the diffusion matrix $\sigma \sigma^{t}(\cdot, \omega)$, there exists a constant $\varepsilon(v, d, \bar{b}, \bar{\sigma}, R, K) \in\left(0, \frac{1}{2}\right)$ such that for all $\omega \in \Omega$,

$$
p_{\omega, U^{x}}(1, x, y) \geqslant \frac{2 \varepsilon}{\left|B_{R}\right|}>0, \quad \text { for all } x \in \mathbb{R}^{d} \text { and } y \in B^{x},
$$

where $\left|B_{R}\right|$ denotes the volume of $B_{R}$. We refer to Corollary A.5 in the appendix for the proof of (2.5).

With the help of (2.5), we are going to use a coupling construction enlarging our probability space $\left(C\left(\mathbb{R}_{+}, \mathbb{R}^{d}\right), \mathscr{F}, \mathrm{P}_{x}^{\omega}\right)$ to include some auxiliary i.i.d. Bernoulli random variables $\left(\lambda_{m}\right)_{m \in \mathbb{N}}$.

Before providing this coupling construction, let us give some other notations. We denote by $\lambda_{j}$ the canonical coordinates on $\{0,1\}^{\mathbb{N}}$ (the variables $\lambda_{j}$ will turn out to be i.i.d. Bernoulli random variables with success probability $\varepsilon$ ). Further, let $\mathscr{S}_{m} \stackrel{\text { def }}{=}$ $\sigma\left\{\lambda_{0}, \ldots, \lambda_{m}\right\}, m \in \mathbb{N}$, denote the canonical filtration on $\{0,1\}^{\mathbb{N}}$ generated by $\left(\lambda_{m}\right)_{m \in \mathbb{N}}$ and $\mathscr{S} \stackrel{\text { def }}{=} \sigma\left\{\bigcup_{m} \mathscr{S}_{m}\right\}$ be the canonical $\sigma$-algebra. To simplify notation let us write for $t \geqslant 0$ :

$$
\mathscr{Z}_{t} \stackrel{\text { def }}{=} \mathscr{F}_{t} \otimes \mathscr{S}_{\lceil t\rceil}, \quad \mathscr{Z} \stackrel{\text { def }}{=} \mathscr{F} \otimes \mathscr{S}=\sigma\left\{\bigcup_{m \in \mathbb{N}} \mathscr{Z}_{m}\right\},
$$

with $\lceil t\rceil \stackrel{\text { def }}{=} \inf \{n \in \mathbb{N}: t \leqslant n\}$. We also introduce the shift operators $\left\{\theta_{m}: m \in \mathbb{N}\right\}$, with $\theta_{m}:\left(C\left(\mathbb{R}_{+}, \mathbb{R}^{d}\right) \times\{0,1\}^{\mathbb{N}}, \mathscr{Z}\right) \rightarrow\left(C\left(\mathbb{R}_{+}, \mathbb{R}^{d}\right) \times\{0,1\}^{\mathbb{N}}, \mathscr{Z}\right)$, such that

$$
\theta_{m}(X ., \lambda .)=\left(X_{m+.}, \lambda_{m+.}\right)
$$

for $X . \in C\left(\mathbb{R}_{+}, \mathbb{R}^{d}\right)$ and $\lambda . \in\{0,1\}^{\mathbb{N}}$.

Now we can state the coupling construction.

THEOREM 2.1 (Coupling construction). - For every $\omega \in \Omega$ and $x \in \mathbb{R}^{d}$ there exists a probability measure $\widehat{\mathrm{P}}_{x}^{\omega}$ on $\left(C\left(\mathbb{R}_{+}, \mathbb{R}^{d}\right) \times\{0,1\}^{\mathbb{N}}, \mathscr{Z}\right)$ depending measurably on $\omega$ and $x$, such that

(1) Under $\widehat{\mathrm{P}}_{x}^{\omega},\left(X_{t}\right)_{t \geqslant 0}$ is $\mathrm{P}_{x}^{\omega}$-distributed, and the $\lambda_{m}, m \geqslant 0$, are i.i.d. Bernoulli variables with success probability $\varepsilon$ (recall (2.5)). 
(2) Under $\widehat{\mathrm{P}}_{x}^{\omega}, \quad \lambda_{m}(m \geqslant 1)$ is independent of $\mathscr{F}_{m} \otimes \mathscr{S}_{m-1}$, and conditioned on $\mathscr{Z}_{m}, X . \circ \theta_{m}$ has the same law as $X$. under $\widehat{\mathrm{P}}_{X_{m}, \lambda_{m}}^{\omega}$, where for $\lambda=0,1, \widehat{\mathrm{P}}_{x, \lambda}^{\omega}$ denotes the law $\widehat{\mathrm{P}}_{x}^{\omega}\left[\cdot \mid \lambda_{0}=\lambda\right]$.

(3) $\widehat{\mathrm{P}}_{x, 1}^{\omega}$ almost surely, $X_{s} \in U^{x}$ for $s \in[0,1]$ (recall (2.1)).

(4) Under $\widehat{\mathrm{P}}_{x, 1}^{\omega}, X_{1}$ is uniformly distributed on $B^{x}$ (recall (2.1)).

Proof. - Given a probability kernel $\widehat{\mathrm{P}}_{x, \lambda}^{\omega}[X . \in O]$, for $O \in \mathscr{F}_{1}, x \in \mathbb{R}^{d}, \lambda \in\{0,1\}$ and $\omega \in \Omega$, there will be a unique probability kernel $\widehat{\mathrm{P}}_{x}^{\omega}$ on $\mathscr{Z}$, for $x \in \mathbb{R}^{d}, \omega \in \Omega$, such that under $\widehat{\mathrm{P}}_{x}^{\omega}$ :

- $\lambda_{m}$ is a Bernoulli random variable with success probability $\varepsilon$, independent of $\mathscr{F}_{m} \otimes \mathscr{S}_{m-1}$, when $m \geqslant 1$

- For $O \in \mathscr{F}_{1}$, the conditional expectation $\widehat{\mathrm{P}}_{x}^{\omega}\left[\theta_{m}^{-1}(X . \in O) \mid \mathscr{Z}_{m}\right] \widehat{\mathrm{P}}_{x}^{\omega}$-a.s. equals $\widehat{\mathrm{P}}_{X_{m}, \lambda_{m}}^{\omega}[O]$.

Here is how we define $\widehat{\mathrm{P}}_{x, \lambda}^{\omega}[X . \in O]$ for $O \in \mathscr{F}_{1}, x \in \mathbb{R}^{d}, \omega \in \Omega$ and $\lambda \in\{0,1\}$, namely we set

$$
\widehat{\mathrm{P}}_{x, \lambda_{0}=1}^{\omega}[X . \in O]=\frac{1}{\left|B_{R}\right|} \int_{B^{x}} d y \mathrm{P}_{x, y}^{\omega, 1}\left[O \mid T_{U^{x}}>1\right],
$$

and

$$
\widehat{\mathrm{P}}_{x, \lambda_{0}=0}^{\omega}[X . \in O]=\frac{1}{1-\varepsilon}\left\{\mathrm{P}_{x}^{\omega}[O]-\frac{\varepsilon}{\left|B_{R}\right|} \int_{B^{x}} d y \mathrm{P}_{x, y}^{\omega, 1}\left[O \mid T_{U^{x}}>1\right]\right\},
$$

where $\mathrm{P}_{x, y}^{\omega, 1}$ is the bridge measure from $x$ to $y$ in time 1 under $\mathrm{P}_{x}^{\omega}$; i.e., $\mathrm{P}_{x, y}^{\omega, 1}$ is the unique probability measure on $\left(C\left([0,1], \mathbb{R}^{d}\right), \mathscr{F}_{1}\right)$ such that for all $O_{s} \in \mathscr{F}_{s}, s<1$ :

$$
\mathrm{P}_{x, y}^{\omega, 1}\left[O_{s}\right]=\frac{1}{p_{\omega}(1, x, y)} \mathrm{E}_{x}^{\omega}\left[O_{s}, p_{\omega}\left(1-s, X_{s}, y\right)\right] .
$$

The proof of the existence of this bridge measure can be found in [31], pp. 137-139. Although the proof in [31] is for the Brownian bridge, it can still be used for the proof of $\mathrm{P}_{x, y}^{\omega, 1}$ with little modification. The only change one need to do is in the proof of (A.8) on p. 138, namely one need to use the inequality $1 / p_{\omega}\left(t-s, X_{s}, y\right) \geqslant$ $\varphi(t-s)^{d / 2} \exp \left\{\frac{\mu\left(X_{s}-y\right)^{2}}{2(t-s)}\right\}, \mu>0, \varphi>0$, which can be found in [8], p. 141.

Observe that $p_{\omega, U^{x}}(1, x, y)=p_{\omega}(1, x, y) \mathrm{P}_{x, y}^{\omega, 1}\left[T_{U^{x}}>1\right]$ and $\mathrm{P}_{x}^{\omega}\left[X . \in O, T_{U^{x}}>1\right.$, $\left.X_{1} \in B^{\prime}\right]=\int_{B^{\prime}} p_{\omega, U^{x}}(1, x, y) \cdot \mathrm{P}_{x, y}^{\omega, 1}\left[X . \in O \mid T_{U^{x}}>1\right] d y$, so in view of (2.5), $\widehat{\mathrm{P}}_{x, \lambda}^{\omega}$ is well defined. It is then straightforward to see that the resulting $\widehat{\mathrm{P}}_{x}^{\omega}$ fulfills (1)-(4).

As a consequence, we have

COROLLARY 2.2 (Markov property). - Under $\widehat{\mathrm{P}}_{x}^{\omega}$, the joint process $\left(X_{m}, \lambda_{m}\right)_{m \in \mathbb{N}}$ is a time homogeneous Markov chain, with respect to the filtration $\left(\mathscr{Z}_{m}=\mathscr{F}_{m} \otimes \mathscr{S}_{m}\right)_{m \in \mathbb{N}}$, and in fact $\widehat{\mathrm{P}}_{x}^{\omega}$-a.s. 


$$
\widehat{\mathrm{P}}_{x}^{\omega}\left[(X ., \lambda .) \circ \theta_{m} \in \star \mid \mathscr{Z}_{m}\right]=\widehat{\mathrm{P}}_{X_{m}, \lambda_{m}}^{\omega}[(X ., \lambda .) \in \star] .
$$

Finally, let us introduce the new annealed measure on $\left(\Omega \times C\left(\mathbb{R}_{+}, \mathbb{R}^{d}\right) \times\{0,1\}^{\mathbb{N}}\right.$, $\mathscr{A} \otimes \mathscr{Z})$, see also (1.8):

$$
\widehat{\mathrm{P}}_{x} \stackrel{\text { def }}{=} \mathbb{P} \times \widehat{\mathrm{P}}_{x}^{\omega} \quad \text { and } \quad \widehat{\mathrm{E}}_{x} \stackrel{\text { def }}{=} \mathbb{E} \times \widehat{\mathrm{E}}_{x}^{\omega},
$$

and observe that by property (1) in Theorem 2.1, $\left(X_{t}\right)_{t \geqslant 0}$ has same distribution under $\widehat{\mathrm{P}}_{x}$ and $\mathrm{P}_{x}$.

\subsection{The regeneration times $\tau_{k}$}

In this part, we will define the regeneration times $\tau_{k}, k \in \mathbb{N}$, and discover the resulting renewal structure.

To define the first regeneration time $\tau_{1}$, we need to introduce a sequence of integervalued $\left(\mathscr{Z}_{t}\right)_{t \geqslant 0}$-stopping times $N_{k}$, for which the condition $\lambda_{N_{k}}=1$ holds, and at these times the process $\left(\ell \cdot X_{s}\right)_{s} \geqslant 0$ reaches essentially a local maximum (within a small variation). Then $\tau_{1}$ is the first $N_{k}+1, k \geqslant 1$, such that the process $\left(\ell \cdot X_{t}\right)_{t \geqslant 0}$ never goes below $\ell \cdot X_{N_{k}+1}-R$ after $N_{k}+1$.

To define $N_{k}$, we introduce the integer-valued $\left(\mathscr{F}_{t}\right)_{t \geqslant 0}$-stopping times $\left(\widetilde{N}_{k}\right)_{k \geqslant 1}$, which are essentially the times when $\left(\ell \cdot X_{s}\right)_{s \geqslant 0}$ reaches local maxima (also within a small variation). Then, we choose $\widetilde{N}_{1}$ to be the first $N_{k}$ with $\lambda_{N_{k}}=1$.

Here is how we precisely define them: first, we introduce for $a>0$ the $\left(\mathscr{F}_{t}\right)_{t \geqslant 0^{-}}$ stopping times $V_{k}(a), k \geqslant 0: V_{0}$ is the first time $\left(\ell \cdot\left(X_{s}-X_{0}\right)\right)_{s \geqslant 0}$ reaches $a$, and $V_{k+1}$ is the first time $\left(\ell \cdot X_{s}\right)_{s \geqslant 0}$ reaches $R$ above the local maximum it reached till $\left\lceil V_{K}\right\rceil$, that is (recall $M(a)$ in (2.3) and $T_{u}$ in (2.2)),

$$
V_{0}(a) \stackrel{\text { def }}{=} T_{a} ; \quad V_{1}(a) \stackrel{\text { def }}{=} T_{M\left(\left\lceil V_{0}(a)\right\rceil\right)+R} ; \quad V_{k+1}(a) \stackrel{\text { def }}{=} T_{M\left(\left[V_{k}(a)\right\rceil\right)+R}
$$

Then, we define $\widetilde{N}_{1}(a)$ to be the first $\left\lceil V_{k}\right\rceil, k \geqslant 0$, such that $\left|\ell \cdot\left(X_{s}-X_{V_{k}}\right)\right| \leqslant \frac{R}{2}$ for all $s \in\left[V_{k},\left\lceil V_{k}\right\rceil\right]$; and $\widetilde{N}_{k+1}(a)$ to be $\widetilde{N}_{1}(3 R)$ shifted after $\widetilde{N}_{k}(a)$ (it is not $\widetilde{N}_{1}(a)$ after $\widetilde{N}_{k}(a)$, the reason for this comes from our definition of $N_{k+1}$ later in (2.15)):

$$
\left\{\begin{array}{l}
\widetilde{N}_{1}(a) \stackrel{\text { def }}{=} \inf \left\{\left\lceil V_{k}(a)\right\rceil: k \geqslant 0, \sup _{s \in\left[V_{k},\left\lceil V_{k}\right\rceil\right]}\left|\ell \cdot\left(X_{s}-X_{V_{k}}\right)\right| \leqslant \frac{R}{2}\right\}, \\
\widetilde{N}_{k+1}(a) \stackrel{\text { def }}{=} \widetilde{N}_{1}(3 R) \circ \theta_{\widetilde{N}_{k}(a)}+\widetilde{N}_{k}(a), \quad k \geqslant 1, \\
N_{1}(a) \stackrel{\text { def }}{=} \inf \left\{\widetilde{N}_{k}(a): k \geqslant 1, \lambda_{\widetilde{N}_{k}(a)}=1\right\} ;
\end{array}\right.
$$

(by convention we set $\widetilde{N}_{k+1}=\infty$ if $\widetilde{N}_{k}=\infty$ ). We illustrate in Fig. 2 the situation, where $\widetilde{N}_{2}(a)$ is $\left\lceil V_{0}(3 R)\right\rceil$ after $\widetilde{N}_{1}(a)$.

Observe that $\widetilde{N}_{k}, k \geqslant 1$, are integer-valued, bigger or equal to 1 , and $\mathrm{P}_{x}^{\omega}$-a.s. $\sup _{s \leqslant \widetilde{N}_{k}} \ell$. $\left(X_{s}-X_{\widetilde{N}_{k}}\right) \leqslant R$, i.e., within a variation of $R, \ell \cdot X_{\widetilde{N}_{k}}$ reaches a local maximum. Now we 


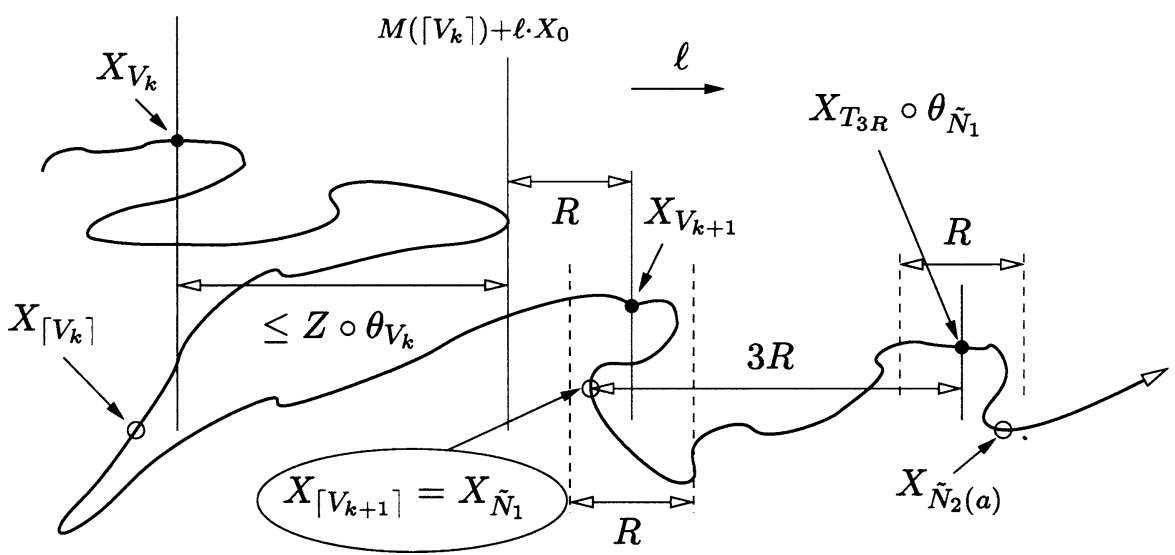

Fig. 2. $V_{k}(a)$ and $\widetilde{N}_{m}(a)$.

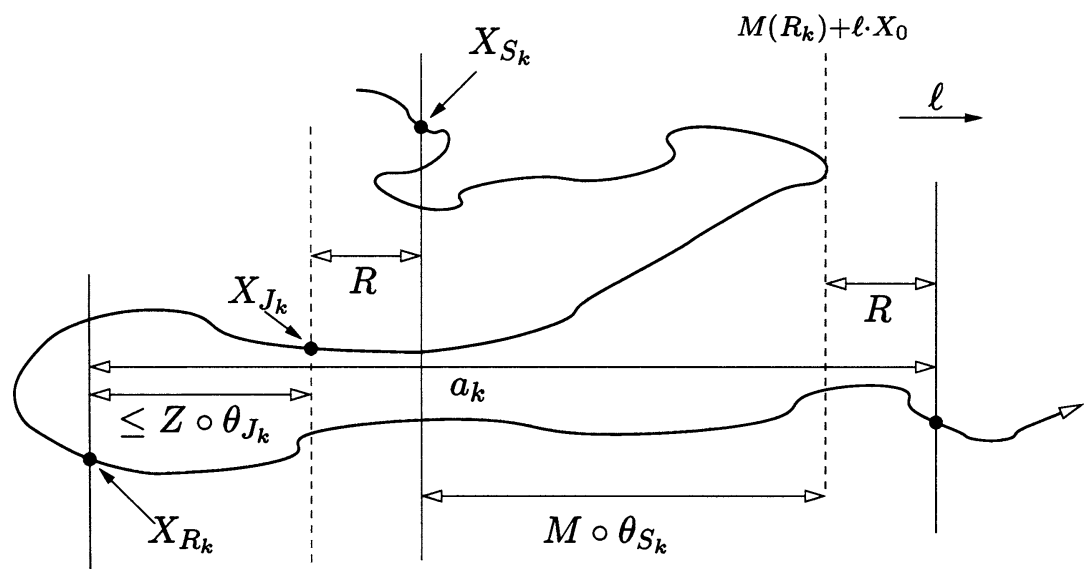

Fig. 3.

can define the $\left(\mathscr{Z}_{t}\right)_{t \geqslant 0}$-stopping times (recall (2.2)):

$$
\left\{\begin{array}{l}
S_{1} \stackrel{\text { def }}{=} N_{1}(3 R)+1 ; \quad J_{1} \stackrel{\text { def }}{=} S_{1}+\widetilde{T}_{-R} \circ \theta_{S_{1}} \\
R_{1} \stackrel{\text { def }}{=}\left\lceil J_{1}\right\rceil=S_{1}+D \circ \theta_{S_{1}}
\end{array}\right.
$$

with $D \stackrel{\text { def }}{=}\left\lceil\widetilde{T}_{-R}\right\rceil$.

Now we shall define the integer-valued $\left(\mathscr{Z}_{t}\right)_{t \geqslant 0}$-stopping time $N_{k+1}, k \geqslant 1$, which is bigger than $R_{k}$ such that $\lambda_{N_{k+1}}=1$, and the process $\left(\ell \cdot X_{s}\right)_{s \geqslant 0}$ does not go above $\ell \cdot X_{N_{k+1}}+R$ until time $N_{k+1}$. More precisely:

$$
N_{k+1} \stackrel{\text { def }}{=} R_{k}+N_{1}\left(a_{k}\right) \circ \theta_{R_{k}} \quad \text { with } a_{k} \stackrel{\text { def }}{=} M\left(R_{k}\right)-\ell \cdot\left(X_{R_{k}}-X_{0}\right)+R,
$$

(the shift $\theta_{R_{k}}$ is not applied to $a_{k}$ in the above definition, cf. Fig. 3). 
The quantity $a_{k}$ in (2.15) is used to make sure that $N_{k+1}$ is an integer bigger than $R_{k}$, such that $\sup _{s \leqslant N_{k+1}} \ell \cdot X_{s} \leqslant \ell \cdot X_{N_{k+1}}+R$ (here is why we defined the stopping times $\left(V_{k}(a)\right)_{k \geqslant 0}$ for a general $\left.a\right)$.

As in (2.14), we define the $\left(\mathscr{Z}_{t}\right)_{t \geqslant 0}$-stopping times:

$$
\left\{\begin{array}{l}
S_{k+1} \stackrel{\text { def }}{=} N_{k+1}+1 ; \quad J_{k+1} \stackrel{\text { def }}{=} S_{k+1}+\widetilde{T}_{-R} \circ \theta_{S_{k+1}} ; \\
R_{k+1} \stackrel{\text { def }}{=}\left\lceil J_{k+1}\right\rceil=S_{k+1}+D \circ \theta_{S_{k+1}} .
\end{array}\right.
$$

Observe that for all $k \in \mathbb{N}$, the $\left(\mathscr{Z}_{t}\right)_{t \geqslant 0}$-stopping times $N_{k}, S_{k}$ and $R_{k}$ are integer-valued, possibly equal to infinity. Of course we have $1 \leqslant N_{1} \leqslant S_{1} \leqslant J_{1} \leqslant R_{1} \leqslant N_{2} \leqslant S_{2} \leqslant J_{2} \leqslant$ $R_{2} \leqslant \cdots \leqslant \infty$.

With the help of these stopping times, the first regeneration time is defined, as in [35], by

$$
\tau_{1} \stackrel{\text { def }}{=} \inf \left\{S_{k}: S_{k}<\infty, R_{k}=\infty\right\} \leqslant \infty .
$$

Again, $\tau_{1}$ is integer-valued, and $\tau_{1} \geqslant 2$, because $N_{1} \geqslant 1$.

With this definition, we see that on the event $\left\{\tau_{1}<\infty\right\}$, $\widehat{\mathrm{P}}_{x}$-a.s., $\ell \cdot X_{s} \leqslant \ell \cdot X_{\tau_{1}-1}+$ $R \leqslant \ell \cdot X_{\tau_{1}}-7 R$, for $s \leqslant \tau_{1}-1$, see also Theorem 2.1 and Fig. 1, i.e. $\left(X_{s}\right)_{s \leqslant \tau_{1}-1}$ remains in the half space $\mathcal{L}\left(\ell \cdot X_{\tau_{1}}-7 R\right)$, with $\mathcal{L}(a) \stackrel{\text { def }}{=}\left\{z \in \mathbb{R}^{d}: z \cdot \ell \leqslant a\right\}$ for $a \in \mathbb{R}$. On the other hand, because the process $\left(\ell \cdot X_{t}\right)_{t \geqslant 0}$ never goes below $\ell \cdot X_{\tau_{1}}-R$ after $\tau_{1}$, i.e. $\left(X_{t}\right)_{t \geqslant \tau_{1}}$ belongs to the half space $\mathcal{R}\left(\ell \cdot X_{\tau_{1}}-R\right)$, where for $a \in \mathbb{R}, \mathcal{R}(a) \stackrel{\text { def }}{=}\left\{z \in \mathbb{R}^{d}: z \cdot \ell \geqslant a\right\}$. This will turn out to be an important issue in the proof of Theorem 2.4.

We will see in Proposition 2.7 below that the $\widehat{P}_{0}$ almost sure finiteness of $\tau_{1}$ is equivalent to $\mathrm{P}_{0}$-a.s., $\lim _{t \rightarrow \infty} \ell \cdot X_{t}=\infty$. For the time being we begin with

LEMmA 2.3. - Suppose that $\widehat{\mathrm{P}}_{0}$-a.s. $\tau_{1}<\infty$, then $\mathrm{P}_{0}[D=\infty]>0$.

Proof. - We prove this by contradiction. If $\mathrm{P}_{0}[D=\infty]=0$, it follows from the stationarity of $\mathbb{P}$-measure that $\int d x \mathrm{P}_{x}[D=\infty]=0$. Thereafter, by Fubini's theorem, there exists a $\mathbb{P}$-null-set $\Upsilon \subset \Omega$, such that for all $\omega \notin \Upsilon$, outside a Lebesgue-null-set $\mathcal{N}(\omega) \subset \mathbb{R}^{d}, \mathrm{P}_{x}^{\omega}[D=\infty]=\mathrm{P}_{x}^{\omega}\left[\widetilde{T}_{-R}=\infty\right]=0$ holds.

Because by our assumptions (1.1), (1.3) and (1.4), the transition density $p_{\omega}(t, x, y)$ exists for all $\omega \in \Omega$ and $t>0$, it follows from the Markov property of $\left(X_{t}\right)_{t \geqslant 0}$ under $\mathrm{P}_{x}^{\omega}$ that for $\omega \notin \Upsilon$ and for all $x \in \mathbb{R}^{d}, \mathrm{P}_{x}^{\omega}\left[\bigcap_{\substack{q \in \mathbb{Q} \\ q>0}} \widetilde{T}_{-R} \circ \theta_{q}<\infty\right]=1$.

Therefore, for $\omega$ outside the $\mathbb{P}$-null-set $\Upsilon$ and all $x \in \mathbb{R}^{d}, \mathrm{P}_{x}^{\omega}\left[\widetilde{T}_{-R / 2}<\infty\right]=1$, which implies by the strong Markov property that $\mathrm{P}_{x}^{\omega}$-a.s. $\liminf _{t} X_{t} \cdot \ell=-\infty$. This contradicts the assumption $\widehat{\mathrm{P}}_{0}\left[\tau_{1}<\infty\right]=1$.

Let us define on the space $\left(\Omega \times C\left(\mathbb{R}_{+}, \mathbb{R}^{d}\right) \times\{0,1\}^{\mathbb{N}}, \mathscr{A} \otimes \mathscr{Z}\right)$ the $\sigma$-algebra $\mathscr{G}$, which is generated by the sets of the form:

$$
\left\{\tau_{1}=m\right\} \cap O_{m-1} \cap\left\{X_{m-1} \cdot \ell>a\right\} \cap\left\{X_{m} \in G\right\} \cap F_{a}, \quad m \geqslant 2, a \in \mathbb{R},
$$

with $O_{m-1} \in \mathscr{Z}_{m-1}, G \subset \mathbb{R}^{d}$ open, and $F_{a} \in \mathscr{H}_{\mathcal{L}(a+R)}$ (recall $\mathscr{H}$ in (1.5) and $\mathcal{L}$ below (2.17)). The situation is shown in Fig. 4. 


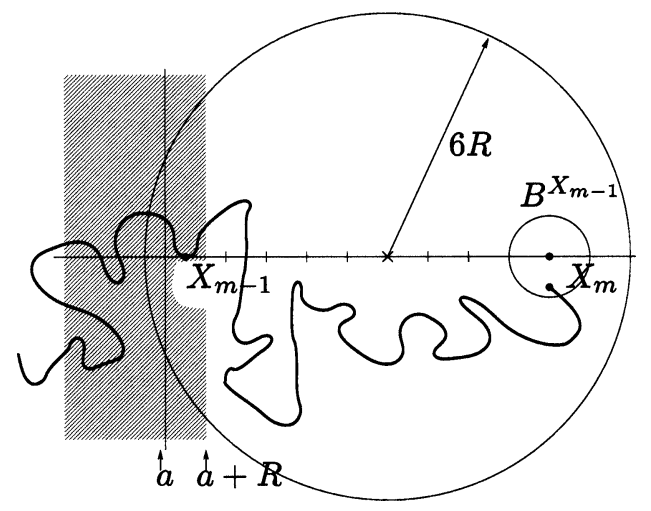

Fig. 4.

Essentially, the $\sigma$-algebra $\mathscr{G}$ describes the history of the Bernoulli variables $\lambda$., the path of the process $\left(X_{t}\right)_{t \geqslant 0}$, and the random environment $\omega$ possibly contributing before time $\tau_{1}-1$.

The key step in the study of the renewal structure mentioned in the introduction is now:

THEOREM 2.4. - Assume that $\widehat{\mathrm{P}}_{0}$-a.s. $\tau_{1}<\infty$. Let $x \in \mathbb{R}^{d}$, and $f, g, h$ be bounded functions, which are respectively $\mathscr{Z}-($ recall $(2.6)), \mathscr{H}_{\mathcal{R}(-R)}-($ recall $\mathscr{H}$ in $(1.5)$ and $\mathcal{R}$ below (2.17)), and $\mathscr{G}$-measurable. Then

$$
\widehat{\mathrm{E}}_{x}\left[f\left(X_{\tau_{1}+\cdot}-X_{\tau_{1}}, \lambda_{\tau_{1}+\cdot}\right) g \circ t_{X_{\tau_{1}}} h\right]=\widehat{\mathrm{E}}_{0}[f(X ., \lambda .) g \mid D=\infty] \cdot \widehat{\mathrm{E}}_{x}[h],
$$

where $t_{y}, y \in \mathbb{R}^{d}$, is the shift operator from the beginning of Section 2.

Proof. - By Lemma 2.3, we know that $\mathrm{P}_{0}[D=\infty]=\widehat{\mathrm{P}}_{0}[D=\infty]>0$, and the righthand side of (2.19) is well-defined.

Since the $\sigma$-algebra $\mathscr{G}$ is generated by sets of the form in (2.18), which form a $\pi$-system, it is sufficient to prove (2.19) for $h=1_{\left\{\tau_{1}=m\right\}} \cdot 1_{\left\{X_{m-1} \cdot \ell>a\right\}} \cdot 1_{F_{a}} \cdot 1_{O_{m-1}} \cdot 1_{X_{m} \in G}$, with $O_{m-1} \in \mathscr{Z}_{m-1}, G \subset \mathbb{R}^{d}$ open, and $F_{a} \in \mathscr{H}_{\mathcal{L}(a+R)}$.

For this special $h$, the left-hand side of (2.19) is now:

$$
\begin{aligned}
\widehat{\mathrm{E}}_{x} & {\left[f\left(X_{\tau_{1}+\cdot}-X_{\tau_{1}}, \lambda_{\tau_{1}+\cdot}\right) g \circ t_{X_{\tau_{1}}} h\right] } \\
& =\widehat{\mathrm{E}}_{x}\left[f\left(X_{m+\cdot}-X_{m}, \lambda_{m+}\right) g \circ t_{X_{m}} ; \tau_{1}=m, O_{m-1}, X_{m-1} \cdot \ell>a, F_{a}, X_{m} \in G\right] .
\end{aligned}
$$

Observe that $\left\{\tau_{1}=m\right\} \cap O_{m-1}=\widetilde{O}_{m-1} \cap\left\{D \circ \theta_{m}=\infty\right\} \cap\left\{\lambda_{m-1}=1\right\}$, for some $\widetilde{O}_{m-1} \in \mathscr{Z}_{m-1} \cap\left\{X_{m-1} \cdot \ell+R \geqslant X_{t} \cdot \ell, \forall t \leqslant m-1\right\}$, therefore the last expression is now:

$$
\begin{gathered}
\mathbb{E}\left\{\widehat { \mathrm { E } } _ { x } ^ { \omega } \left[\widehat{\mathrm{E}}_{x}^{\omega}\left[f\left(X_{m+\cdot}-X_{m}, \lambda_{m+.}\right) g \circ t_{X_{m}} ; X_{m} \in G, D \circ \theta_{m}=\infty \mid \mathscr{Z}_{m-1}\right]\right.\right. \\
\left.\left.F_{a}, \widetilde{O}_{m-1}, X_{m-1} \cdot \ell>a, \lambda_{m-1}=1\right]\right\} .
\end{gathered}
$$

By the Markov property, cf. (2.10), we observe that $\mathrm{P}_{x}^{\omega}$-a.s. on the event $\left\{\lambda_{m-1}=1\right\}$,

$$
\widehat{\mathrm{E}}_{x}^{\omega}\left[f\left(X_{m+\cdot}-X_{m}, \lambda_{m+.}\right) g \circ t_{X_{m}} ; X_{m} \in G, D \circ \theta_{m}=\infty \mid \mathscr{Z}_{m-1}\right]
$$




$$
\begin{aligned}
& =\widehat{\mathrm{E}}_{X_{m-1}, 1}^{\omega}\left[f\left(X_{1+}-X_{1}, \lambda_{1+.}\right) g \circ t_{X_{1}} ; X_{1} \in G, D \circ \theta_{1}=\infty\right] \\
& =\widehat{\mathrm{E}}_{X_{m-1}, 1}^{\omega}\left[\widehat{\mathrm{E}}_{X_{1}, \lambda_{1}}^{\omega}\left[f\left(X .-X_{0}, \lambda .\right) g \circ t_{X_{0}} ; D=\infty\right], X_{1} \in G\right] .
\end{aligned}
$$

Note that, by Theorem 2.1, $\lambda_{1}$ is independent of $X_{1}$ under the measure $\widehat{\mathrm{P}}_{y, 1}^{\omega}$, for all $y \in \mathbb{R}^{d}$; and using property (4) of Theorem 2.1 , the last expression is:

$$
\frac{1}{\left|B_{R}\right|} \int_{B^{X_{m-1} \cap G}} d y \widehat{\mathrm{E}}_{y}^{\omega}\left[f(X .-y, \lambda .) g \circ t_{y}, D=\infty\right] .
$$

Plugging this formula into (2.20) and using Fubini's theorem, the left-hand side of (2.19) now equals

$$
\begin{aligned}
\frac{1}{\left|B_{R}\right|} \int d y \mathbb{E}\left\{\widehat { \mathrm { E } } _ { x } ^ { \omega } \left[\widehat{\mathrm{E}}_{y}^{\omega}\left[f(X .-y, \lambda .) g \circ t_{y}, D=\infty\right] ;\right.\right. \\
\left.\left.F_{a}, \widetilde{O}_{m-1}, X_{m-1} \cdot \ell>a, \lambda_{m-1}=1,\left\{y \in B^{X_{m-1}} \cap G\right\}\right]\right\} .
\end{aligned}
$$

Set $V \stackrel{\text { def }}{=}\left\{F_{a}, \widetilde{O}_{m-1}, X_{m-1} \cdot \ell>a, \lambda_{m-1}=1, y \in B^{X_{m-1}} \cap G\right\}$, the last expression equals

$$
\frac{1}{\left|B_{R}\right|} \int d y \mathbb{E}\left\{\widehat{\mathrm{P}}_{x}^{\omega}[V] \cdot \widehat{\mathrm{E}}_{y}^{\omega}[f(X .-y, \lambda .), D=\infty] \cdot g \circ t_{y}\right\}
$$

Observe that $1_{\left\{y \in B^{X_{m-1}}\right.}$ is zero for $y \cdot \ell-8 R \leqslant X_{m-1} \cdot \ell$, see also Fig. 4. Therefore, in the above integral we only need to consider $y$ such that $a<y \cdot \ell-8 R$, and thus $F_{a} \in \mathscr{H}_{\mathcal{L}(y \cdot \ell-7 R)}$. Also observe that for the $\widetilde{O}_{m-1}$ introduced above (2.20), we have $\widetilde{O}_{m-1} \subset\left\{X_{m-1} \cdot \ell+R \geqslant X_{t} \cdot \ell, \forall t \leqslant m-1\right\}$. Therefore, we see that $\widehat{\mathrm{P}}_{x}^{\omega}[V]$ is $\mathscr{H}_{\mathcal{L}(y \cdot \ell-7 R)^{-}}$ measurable.

On the other hand, since $g$ is $\mathscr{H}_{\mathcal{R}(-R)}$-measurable and due to the restriction $D=\infty$, we observe that $\widehat{\mathrm{E}}_{y}^{\omega}[f(X .-y, \lambda),. D=\infty] \cdot g \circ t_{y}$ is $\mathscr{H}_{\mathcal{R}(y \cdot \ell-R)}$-measurable.

As a result of $R$-separation, cf. (1.6), we see that $\widehat{\mathrm{P}}_{x}^{\omega}[V]$ and $\widehat{\mathrm{E}}_{y}^{\omega}[f(X .-y, \lambda$.), $D=$ $\infty] \cdot g \circ t_{y}$ are independent under the $\mathbb{P}$-measure. Using this observation, (2.21) equals

$$
\begin{aligned}
\int d y \widehat{\mathrm{E}}_{x}\left[\frac{1_{V}}{\left|B_{R}\right|}\right] \cdot \widehat{\mathrm{E}}_{y}\left[f(X .-y, \lambda .) g \circ t_{y}, D=\infty\right] \\
=\left(\int d y \widehat{\mathrm{E}}_{x}\left[\frac{1_{V}}{\left|B_{R}\right|}\right]\right) \cdot \widehat{\mathrm{E}}_{0}[f(X ., \lambda .) g, D=\infty],
\end{aligned}
$$

where we used the stationarity of the $\mathbb{P}$-measure in the last step. By taking $f=g=1$, we get from the above calculation that $\widehat{\mathrm{E}}_{x}[h]=\widehat{\mathrm{P}}_{0}[D=\infty] \cdot \int d y \widehat{\mathrm{E}}_{x}\left[1_{V} /\left|B_{R}\right|\right]$, therefore the left-hand side of (2.19) is now

$$
\widehat{\mathrm{E}}_{0}[f(X ., \lambda .) g, D=\infty] \cdot \frac{\widehat{\mathrm{E}}_{x}[h]}{\widehat{\mathrm{P}}_{0}[D=\infty]}=\widehat{\mathrm{E}}_{0}[f(X ., \lambda .) g \mid D=\infty] \cdot \widehat{\mathrm{E}}_{x}[h] .
$$

This finishes the proof. 
We now define inductively on the event $\left\{\tau_{1}<\infty\right\}$ a non-decreasing sequence of random variables, by viewing $\tau_{k}, k \geqslant 1$, as a function of $\left(X ., \lambda_{\text {. }}\right)$ :

$$
\tau_{k+1}\left(\left(X ., \lambda_{.}\right)\right) \stackrel{\text { def }}{=} \tau_{1}\left(\left(X ., \lambda_{.}\right)\right)+\tau_{k}\left(\left(X_{\tau_{1}+\cdot}-X_{\tau_{1}}, \lambda_{\tau_{1}+.}\right)\right), \quad k \geqslant 1,
$$

and set by convention $\tau_{k+1}=\infty$ on $\left\{\tau_{k}=\infty\right\}$. We observe that for each $k, \tau_{k}$ is either infinite or a positive integer. Of course, $\tau_{k+1}=\tau_{k}\left(\left(X ., \lambda_{.}\right)\right)+\tau_{1}\left(\left(X_{\tau_{k}+\cdot}-X_{\tau_{k}}, \lambda_{\tau_{k}+.}\right)\right)$, but we prefer the definition (2.22) in view of the proof of the renewal structure promised in the introduction (in the next theorem, we set to $\tau_{0}=0$ ):

Theorem 2.5 (Renewal structure). - Assume that $\widehat{\mathrm{P}}_{0}$-a.s., $\tau_{1}<\infty$. Then under the measure $\widehat{\mathrm{P}}_{0}$, the random variables $Z_{k} \stackrel{\text { def }}{=}\left(X_{\left(\tau_{k}+\right) \wedge\left(\tau_{k+1}-1\right)}-X_{\tau_{k}} ; X_{\tau_{k+1}}-X_{\tau_{k}} ; \tau_{k+1}-\tau_{k}\right)$, $k \geqslant 0$, are independent. Furthermore, $Z_{k}, k \geqslant 1$, under $\widehat{\mathrm{P}}_{0}$, have the distribution of $Z_{0}=\left(X_{\cdot \wedge\left(\tau_{1}-1\right)}-X_{0} ; X_{\tau_{1}}-X_{0} ; \tau_{1}\right)$ under $\widehat{\mathrm{P}}_{0}[\cdot \mid D=\infty]$.

Proof. - Let us define on the space $\left(\Omega \times C\left(\mathbb{R}_{+}, \mathbb{R}^{d}\right) \times\{0,1\}^{\mathbb{N}}, \mathscr{A} \otimes \mathscr{Z}\right)$ the $\sigma$-algebra $\mathscr{G}_{n+1}$, which is generated by $\left(Z_{k}\right)_{0 \leqslant k \leqslant n}$. It suffices to show that for $h$ bounded and $\mathscr{G}_{n+1^{-}}$measurable, $n \geqslant 0$,

$$
\widehat{\mathrm{E}}_{0}\left[h, Z_{n+1} \in *\right]=\widehat{\mathrm{E}}_{0}[h] \cdot \widehat{\mathrm{P}}_{0}\left[Z_{0} \in * \mid D=\infty\right] .
$$

We prove this by induction. The case $n=0$ follows from Theorem 2.4, because $\mathscr{G}_{1} \subset \mathscr{G}$, with $\mathscr{G}$ defined in (2.18). For the step $n \rightarrow n+1$, we observe that because $\mathscr{G}_{n+1}$ is generated by $\mathscr{G}_{1}$ and $\theta_{\tau_{1}}^{-1}\left(\mathscr{G}_{n}\right)$, without loss of generality we can assume that $h=h_{1} \cdot h_{n} \circ \theta_{\tau_{1}}$, with $h_{n} \in \mathscr{G}_{n}$ and $h_{1} \in \mathscr{G}_{1}$. It follows from Theorem 2.4 that the lefthand side of (2.23) equals

$$
\widehat{\mathrm{E}}_{0}\left[\left(h_{n} 1_{\left\{Z_{n} \in *\right\}}\right) \circ \theta_{\tau_{1}} \cdot h_{1}\right]=\widehat{\mathrm{E}}_{0}\left[h_{n} 1_{\left\{Z_{n} \in *\right\}} ; \quad D=\infty\right] \cdot \frac{\widehat{\mathrm{E}}_{0}\left[h_{1}\right]}{\mathrm{P}_{0}[D=\infty]} .
$$

Observe that $\{D=\infty\}=\left\{\widetilde{T}_{-R}=\infty\right\}=\left\{\widetilde{T}_{-R} \geqslant \tau_{1}\right\}=\left\{D \geqslant \tau_{1}\right\}$ (the equalities hold $\widehat{\mathrm{P}}_{0}$-a.s.). Indeed, we only need to show the last equality: from the definition of $D$, it is obvious that $\left\{\widetilde{T}_{-R} \geqslant \tau_{1}\right\} \subset\left\{D \geqslant \tau_{1}\right\}$; to the opposite direction, we see that $D \geqslant \tau_{1}$ implies $\widetilde{T}_{-R}>\tau_{1}-1$, and in addition because $\left(X_{N_{j}}-X_{0}\right) \cdot \ell \geqslant 3 R$ for all $j \geqslant 1$, cf. (2.14), and $\widetilde{T}_{-R} \circ \theta_{\tau_{1}}=\infty, \widetilde{T}_{-R}=\infty$ follows. Then, we observe that up-to a $\widehat{\mathrm{P}}_{0}$-null-set, $\left\{D \geqslant \tau_{1}\right\}$ lies in $\mathscr{G}_{1}$ (indeed, $\widehat{\mathrm{P}}_{0}$-a.s. $\left\{D \geqslant \tau_{1}=m\right\}=\{D>m-1\} \cap$ $\left\{\tau_{1}=m\right\}$, thus by (2.18), the claim follows), therefore $h_{n} \cdot 1_{\{D=\infty\}} \in \mathscr{G}_{n}$. Hence, it follows by the induction assumption that the right-hand side of the previous expression equals

$$
\begin{gathered}
\widehat{\mathrm{P}}_{0}\left[Z_{0} \in * \mid D=\infty\right] \cdot \widehat{\mathrm{E}}_{0}\left[h_{n} ; D=\infty\right] \cdot \frac{\widehat{\mathrm{E}}_{0}\left[h_{1}\right]}{\mathrm{P}_{0}[D=\infty]} \\
=\widehat{\mathrm{P}}_{0}\left[Z_{0} \in * \mid D=\infty\right] \cdot \widehat{\mathrm{E}}_{0}\left[h_{1} h_{n} \circ \theta_{\tau_{1}}\right] .
\end{gathered}
$$

This finishes the proof.

Remark 2.6. - In the above theorem, the renewal structure is proved for the trajectory between times $\tau_{k}$ and $\tau_{k+1}-1$, unlike in [35]. Nevertheless, we have very good control 
over the trajectory between times $\tau_{k}$ and $\tau_{k+1}$, because by our construction $\lambda_{\tau_{k+1}-1}=1$, hence, $\widehat{P}_{0}$-a.s. $X_{s} \in B^{X_{\tau_{k+1}-1}}$, for all $s \in\left[\tau_{k+1}-1, \tau_{k+1}\right]$. I.e., the path between $\tau_{k+1}-1$ and $\tau_{k+1}$ remains in a ball of radius $6 R$, see also Fig. 4 .

Proposition 2.7. $-\widehat{\mathrm{P}}_{0}$-a.s. $\tau_{1}<\infty$ if and only if $\mathrm{P}_{0}$-a.s. $\lim _{t \rightarrow \infty} X_{t} \cdot \ell=\infty$.

Proof. - If $\widehat{\mathrm{P}}_{0}$-a.s. $\tau_{1}<\infty$, then it follows from Theorem 2.5 that $\widehat{\mathrm{P}}_{0}$-a.s. $\tau_{m}<\infty$, for all $m \geqslant 1$, and by definition of $\tau_{m}$ that $\widehat{\mathrm{P}}_{0}$-a.s. $\lim _{m \rightarrow \infty} X_{\tau_{m}} \cdot \ell=\infty$. Therefore, $\lim _{t \rightarrow \infty} X_{t} \cdot \ell=\infty$.

To show the opposite direction, we first claim that $\widehat{\mathrm{P}}_{0}$-a.s. $N_{1}<\infty$, and hence $S_{1}<\infty$. Let us define

$$
Z \stackrel{\text { def }}{=} \sup _{s \leqslant 1}\left|X_{s}-X_{0}\right| \quad \text { and } \quad A \stackrel{\text { def }}{=}\left\{Z>\frac{R}{2}\right\}
$$

and observe that because of the assumption (1.1) and (1.4) it follows from the Support Theorem of Stroock-Varadhan, cf. [2], p. 25, that there exists a constant $c_{0}(K, \bar{b}, \bar{\sigma}, \nu, R, d)>0$ such that for all $x \in \mathbb{R}^{d}$ and $\omega \in \Omega$ :

$$
\mathrm{P}_{x}^{\omega}\left[A^{c}\right] \geqslant c_{0}>0
$$

Since $\lim _{t \rightarrow \infty} X_{t} \cdot \ell=\infty, \mathrm{P}_{0}$-a.s., we see that there exists a $\mathbb{P}$-null-set $\Upsilon \subset \Omega$ such that for all $\omega \notin \Upsilon, \mathrm{P}_{0}^{\omega}$-a.s. $V_{k}(3 R)<\infty$ for all $k \in \mathbb{N}$, cf. (2.12) for the definition of $V_{k}$. Let us define

$$
A_{k} \stackrel{\text { def }}{=}\left\{\sup _{s \in\left[V_{k},\left\lceil V_{k}\right\rceil\right]}\left|\ell \cdot\left(X_{s}-X_{V_{k}}\right)\right|>\frac{R}{2}\right\}, \quad k \geqslant 0,
$$

then it follows from induction and the strong Markov property that for $n \in \mathbb{N}$ and $\omega \notin \Upsilon, \mathrm{P}_{0}^{\omega}\left[\bigcap_{0 \leqslant k \leqslant n} A_{k}\right] \leqslant\left(1-c_{0}\right)^{n}$. As a result, for all $\omega \notin \Upsilon, \mathrm{P}_{0}^{\omega}\left[\widetilde{N}_{1}(3 R)=\infty\right] \leqslant$ $\mathrm{P}_{0}^{\omega}\left[\bigcap_{k \geqslant 0} A_{k}\right]=0$. By the stationarity of $\mathbb{P}$-measure, we see that $\mathrm{P}_{x}$-a.s. $\widetilde{N}_{1}<\infty$, for all $x \in \mathbb{R}^{d}$. Therefore, $\int d x \mathrm{P}_{x}\left[\widetilde{N}_{1}=\infty\right]=0$, so it follows from Fubini's theorem that there is a $\mathbb{P}$-null-set $\Psi \subset \Omega$, such that for all $\omega \notin \Psi$, outside a Lebesgue-null-set $\mathcal{N}(\omega) \subset \mathbb{R}^{d}, \mathrm{P}_{x}^{\omega}\left[\widetilde{N}_{1}=\infty\right]=0$. Using the positivity of $p_{\omega}(n, y, z)$, with a somewhat similar argument as in the last two paragraphs of the proof of Lemma 2.3, we see by induction that $\mathrm{P}_{0}\left[\widetilde{N}_{m}=\infty\right]=0$, for $m \geqslant 1$.

Clearly, for arbitrary $n \geqslant 1, \widehat{\mathrm{P}}_{0}\left[N_{1}(3 R)=\infty\right] \leqslant \widehat{\mathrm{P}}_{0}\left[\lambda_{\widetilde{N}_{m}(3 R)}=0, \forall m \leqslant n\right] \leqslant(1-\varepsilon)^{n}$ holds. As a result, $\widehat{\mathrm{P}}_{0}$-a.s. $N_{1}<\infty$.

We now can prove that $\widehat{\mathrm{P}}_{0}$-a.s. $\tau_{1}<\infty$. To show this we note that by similar computations as in the proof of Theorem 2.4 (see (2.20), (2.21)):

$$
\begin{aligned}
\widehat{\mathrm{P}}_{0} & {\left[R_{k}<\infty\right]=\mathbb{E}\left[\widehat{\mathrm{P}}_{0}^{\omega}\left[N_{k}<\infty, D \circ \theta_{N_{k}+1}<\infty\right]\right] } \\
& =\sum_{m \geqslant 2} \mathbb{E}\left[\widehat{\mathrm{P}}_{0}^{\omega}\left[N_{k}=m-1, D \circ \theta_{m}<\infty\right]\right] \\
& =\sum_{m \geqslant 2} \mathbb{E}\left[\widehat{\mathrm{P}}_{0}^{\omega}\left[N_{k}=m-1, \widehat{\mathrm{P}}_{X_{m-1}, 1}^{\omega}\left[\widehat{\mathrm{P}}_{X_{1}, \lambda_{1}}^{\omega}[D<\infty]\right]\right]\right] \\
& =\sum_{m \geqslant 2} \frac{1}{\left|B_{R}\right|} \int d y \mathbb{E}\left[\widehat{\mathrm{P}}_{0}^{\omega}\left[\Gamma, \lambda_{m-1}=1, y \in B^{X_{m-1}}\right] \cdot \widehat{\mathrm{P}}_{y}^{\omega}[D<\infty]\right],
\end{aligned}
$$


for some $\Gamma \in \mathscr{F}_{m-1} \otimes \mathscr{S}_{m-2}$ such that $\left\{N_{k}=m-1\right\}=\Gamma \cap\left\{\lambda_{m-1}=1\right\}$. We observe that $\Gamma \subset\left\{X_{m-1} \cdot \ell+R \geqslant X_{t} \cdot \ell, \forall t \leqslant m-1\right\}$, hence as in the proof of Theorem 2.4, $\widehat{\mathrm{P}}_{0}^{\omega}\left[\Gamma, y \in B^{X_{m-1}}, \lambda_{m-1}=1\right]$ and $\widehat{\mathrm{P}}_{y}^{\omega}[D<\infty]$ are $\mathbb{P}$-independent, therefore the last expression equals

$$
\begin{aligned}
& \sum_{m \geqslant 2} \frac{1}{\left|B_{R}\right|} \int d y \widehat{\mathrm{P}}_{0}\left[\Gamma, \lambda_{m-1}=1, y \in B^{X_{m-1}}\right] \cdot \mathrm{P}_{0}[D<\infty] \\
& \quad=\widehat{\mathrm{P}}_{0}\left[S_{k}<\infty\right] \cdot \mathrm{P}_{0}[D<\infty] \leqslant \widehat{\mathrm{P}}_{0}\left[R_{k-1}<\infty\right] \cdot \mathrm{P}_{0}[D<\infty],
\end{aligned}
$$

(it is not hard to see that the last inequality above is indeed an equality) so by induction we obtain that

$$
\widehat{\mathrm{P}}_{0}\left[R_{k}<\infty\right] \leqslant \mathrm{P}_{0}[D<\infty]^{k} .
$$

On the other hand, as in the proof of Lemma 2.3, $\mathrm{P}_{0}$-a.s. $\lim _{t} X_{t} \cdot \ell=\infty$ implies $\mathrm{P}_{0}[D=$ $\infty]>0$. Therefore, from (2.27) and $\widehat{\mathrm{P}}_{0}$-a.s. $S_{1}<\infty, S_{k+1}<\infty$ on $\left\{R_{k}<\infty\right\}$ we see that $\widehat{\mathrm{P}}_{0}$-a.s. $\inf \left\{k \geqslant 1: S_{k}<\infty, R_{k}=\infty\right\}<\infty$, which proves $\widehat{\mathrm{P}}_{0}$-a.s. $\tau_{1}<\infty$.

\section{Law of large numbers and central limit theorem}

In this section we will provide a sufficient condition to derive a strong law of large numbers and a functional central limit theorem. Some parts of the proofs presented in this section are similar to the proofs of Theorem 2.3 on p. 1864 in [35], and of Theorem 4.1 on pp. 130-131 in [32]. We will also use some classical results about continuous martingales, which are presented in the appendix.

We begin with

LEMMA 3.1. - Under (3.1-i), (3.2-i) holds:

$$
\begin{aligned}
& \mathrm{P}_{0} \text {-a.s. } \quad \lim _{t \rightarrow \infty} \ell \cdot X_{t}=\infty \text { and } \widehat{\mathrm{E}}_{0}\left[\tau_{1} \mid D=\infty\right]<\infty, \\
& \widehat{\mathrm{E}}_{0}\left[\left|X_{\tau_{1}}\right| \mid D=\infty\right]<\infty .
\end{aligned}
$$

Analogously, under (3.1-ii), (3.2-ii) holds:

$$
\begin{aligned}
& \mathrm{P}_{0} \text {-a.s. } \quad \lim _{t \rightarrow \infty} \ell \cdot X_{t}=\infty \text { and } \widehat{\mathrm{E}}_{0}\left[\tau_{1}^{2} \mid D=\infty\right]<\infty \\
& \widehat{\mathrm{E}}_{0}\left[\left|X_{\tau_{1}}\right|^{2} \mid D=\infty\right]<\infty .
\end{aligned}
$$

Proof. - First, we prove the implication (3.1-ii) $\Rightarrow$ (3.2-ii). From Lemma 2.3 and Proposition 2.7 we see that $\mathrm{P}_{0}[D=\infty]>0$, and hence $\widehat{\mathrm{E}}_{x}\left[\tau_{1}^{2} \mid D=\infty\right]$ is well-defined. Further, because $\tau_{1}$ only takes integer value bigger or equal to 2 , we can write

$$
\widehat{\mathrm{E}}_{0}\left[\left|X_{\tau_{1}}\right|^{2} \mid D=\infty\right]=\sum_{n=2}^{\infty} \widehat{\mathrm{E}}_{0}\left[\left|X_{n}\right|^{2}, \tau_{1}=n \mid D=\infty\right] .
$$

Observe that $\mathrm{P}_{0}^{\omega}$-a.s. (and therefore $\widehat{\mathrm{P}}_{0}^{\omega}$-a.s.)

$$
\left|X_{n}\right|^{2}=\left|\int_{0}^{n} b\left(X_{s}, \omega\right) d s+\int_{0}^{n} \sigma\left(X_{s}, \omega\right) d W_{s}\right|^{2} \leqslant 2 \bar{b}^{2} n^{2}+2\left|Y_{n}(\omega)\right|^{2},
$$


where $W_{s}$ is some suitable $\mathscr{F}_{t}$ Brownian motion, $\bar{b}$ appears in (1.1), and

$$
Y_{t}(\omega):=\int_{0}^{t} \sigma\left(X_{s}, \omega\right) d W_{s}
$$

is an $\left(\mathscr{F}_{t}\right)_{t \geqslant 0}$ local martingale under $\mathrm{P}_{0}^{\omega}$. Thus, the right-hand side of (3.3) is

$$
\leqslant 2 \bar{b}^{2} \widehat{\mathrm{E}}_{0}\left[\tau_{1}^{2} \mid D=\infty\right]+2 \sum_{n=2}^{\infty} \widehat{\mathrm{E}}_{0}\left[\left|Y_{n}\right|^{2}, \tau_{1}=n \mid D=\infty\right] .
$$

By Hölder's inequality with $p, q \geqslant 1$ such that $\frac{1}{p}+\frac{1}{q}=1$, each term in the summation of the last display can be estimated by

$$
\begin{aligned}
& \widehat{\mathrm{E}}_{0}\left[\left|Y_{n}\right|^{2}, \tau_{1}=n \mid D=\infty\right] \leqslant \widehat{\mathrm{E}}_{0}\left[\left|Y_{n}\right|^{2 p} \mid D=\infty\right]^{1 / p} \cdot \widehat{\mathrm{P}}_{0}\left[\tau_{1}=n \mid D=\infty\right]^{1 / q} \\
& \quad \leqslant \frac{1}{\widehat{\mathrm{P}}_{0}[D=\infty]^{1 / p}} \widehat{\mathrm{E}}_{0}\left[\left|Y_{n}\right|^{2 p}\right]^{1 / p} \cdot \widehat{\mathrm{P}}_{0}\left[\tau_{1}=n \mid D=\infty\right]^{1 / q} .
\end{aligned}
$$

From the assumption (1.4), we see that $\left\langle Y^{i}(\omega)\right\rangle_{t} \leqslant v t$ for all $\omega \in \Omega, i=1, \ldots, d$, so we can apply (A.1) in the appendix and obtain that the rightmost side of the above expression is smaller than

$$
\frac{c(p, d, v)}{\widehat{\mathrm{P}}_{0}[D=\infty]^{1 / p}} \cdot n \widehat{\mathrm{P}}_{0}\left[\tau_{1}=n \mid D=\infty\right]^{1 / q} .
$$

Coming back to (3.3), we see that in order to show $\widehat{\mathrm{E}}_{0}\left[\left|X_{\tau_{1}}\right|^{2} \mid D=\infty\right]<\infty$, it suffices to prove $\sum_{n=2}^{\infty} n \widehat{\mathrm{P}}_{0}\left[\tau_{1}=n \mid D=\infty\right]^{1 / q}<\infty$, for some $q>1$.

To this end, observe that by assumption (3.1-ii), $\widehat{\mathrm{E}}_{0}\left[\tau_{1}^{2} \mid D=\infty\right]=\sum_{n=2}^{\infty} n^{2} \widehat{\mathrm{P}}_{0}\left[\tau_{1}=\right.$ $n \mid D=\infty]<\infty$, and hence with Hölder's inequality:

$$
\begin{gathered}
\sum_{n} n \widehat{\mathrm{P}}_{0}\left[\tau_{1}=n \mid D=\infty\right]^{1 / q}=\sum_{n} n^{1-2 / q} n^{2 / q} \widehat{\mathrm{P}}_{0}\left[\tau_{1}=n \mid D=\infty\right]^{1 / q} \\
\leqslant\left(\sum_{n} n^{(1-2 / q) p}\right)^{1 / p} \cdot\left(\sum_{n} n^{2} \widehat{\mathrm{P}}_{0}\left[\tau_{1}=n \mid D=\infty\right]\right)^{1 / q}<\infty,
\end{gathered}
$$

provided $q$ close to 1 , i.e. $p$ close to $\infty$.

For the implication (3.1-i) $\Rightarrow$ (3.2-i), we proceed similarly as above. Instead of (3.6), we use

$$
\begin{aligned}
& \sum_{n} \sqrt{n} \widehat{\mathrm{P}}_{0}\left[\tau_{1}=n \mid D=\infty\right]^{1 / q} \\
& \leqslant\left(\sum_{n} n^{(1 / 2-1 / q) p}\right)^{1 / p} \cdot\left(\sum_{n} n \widehat{\mathrm{P}}_{0}\left[\tau_{1}=n \mid D=\infty\right]\right)^{1 / q}<\infty,
\end{aligned}
$$

for $q$ close to 1 . This completes the proof.

Now we are ready to prove the strong law of large numbers: 
THEOREM 3.2 (Strong law of large numbers). - Assume (3.1-i), then

$$
\mathrm{P}_{0} \text {-a.s. } \frac{X_{t}}{t} \stackrel{t \rightarrow \infty}{\longrightarrow} v \stackrel{\text { def }}{=} \frac{\widehat{\mathrm{E}}_{0}\left[X_{\tau_{1}} \mid D=\infty\right]}{\widehat{\mathrm{E}}_{0}\left[\tau_{1} \mid D=\infty\right]},
$$

and $\ell \cdot v>0$.

Proof. - Because $X$. has same distribution under $\widehat{\mathrm{P}}_{0}$ and $\mathrm{P}_{0}$, it is sufficient to show that $\widehat{\mathrm{P}}_{0}$-a.s. $\frac{X_{t}}{t} \stackrel{t \rightarrow \infty}{\longrightarrow} v$.

Further, from our construction of $S_{k}$ and $\tau_{1}$, see (2.14), (2.16) and (2.17), it is clear that $\widehat{\mathrm{P}}_{0}$-a.s. $X_{\tau_{1}} \cdot \ell>0$, thus $\ell \cdot v>0$ is immediate.

By Theorem 2.5, the strong law of large numbers applied on the i.i.d. random variables $\left(\tau_{n+1}-\tau_{n}, X_{\tau_{n+1}}-X_{\tau_{n}}\right), n \geqslant 1$, shows that $\widehat{\mathrm{P}}_{0}$-a.s.

$$
\frac{X_{\tau_{n}}}{n} \stackrel{n \rightarrow \infty}{\longrightarrow} \widehat{\mathrm{E}}_{0}\left[X_{\tau_{1}} \mid D=\infty\right], \quad \frac{\tau_{n}}{n} \stackrel{n \rightarrow \infty}{\longrightarrow} \widehat{\mathrm{E}}_{0}\left[\tau_{1} \mid D=\infty\right] .
$$

For each $t>0$, we define a non-decreasing integer-valued function $k(t)$, which tends to infinity $\widehat{P}_{0}$-a.s., such that

$$
\left.\tau_{k(t)} \leqslant t<\tau_{k(t)+1} \quad \text { (with the convention } \tau_{0}=0\right) .
$$

Dividing the above inequality by $k(t)$ and using (3.8), we find that $\widehat{\mathrm{P}}_{0}$-a.s.

$$
\frac{k(t)}{t} \stackrel{t \rightarrow \infty}{\longrightarrow} \frac{1}{\widehat{\mathrm{E}}_{0}\left[\tau_{1} \mid D=\infty\right]}
$$

Further we observe that, because of $X_{t} / t=X_{\tau_{k(t)}} / t+\left(X_{t}-X_{\tau_{k(t)}}\right) / t$, and in view of (3.8) and (3.10), $\widehat{\mathrm{P}}_{0}$-a.s. $X_{\tau_{k(t)}} / t \stackrel{t \rightarrow \infty}{\longrightarrow}\left(\widehat{\mathrm{E}}_{0}\left[X_{\tau_{1}} \mid D=\infty\right]\right) /\left(\widehat{\mathrm{E}}_{0}\left[\tau_{1} \mid D=\infty\right]\right)$, we can show (3.7) by proving $\widehat{\mathrm{P}}_{0}$-a.s. $\left(X_{t}-X_{\tau_{k(t)}}\right) / t \stackrel{t \rightarrow \infty}{\longrightarrow} 0$.

To prove this, we observe that since $X$. is the solution of the stochastic differential equation (1.7), we have $\widehat{P}_{0}$-a.s.

$$
\frac{1}{t}\left|X_{t}-X_{\tau_{k(t)}}\right| \leqslant \bar{b} \frac{\left|t-\tau_{k(t)}\right|}{t}+\frac{2}{t} \sup _{s \leqslant t}\left|Y_{s}\right|,
$$

with the $\left(\mathscr{F}_{t}\right)_{t \geqslant 0}$ local martingale $Y_{t}(\omega)$ defined in (3.4). In view of (3.9) and (3.10), the first term in the last expression tends to zero $\widehat{P}_{0}$-a.s. Applying (A.2), the second term $\mathrm{P}_{0}^{\omega}$-a.s. tends to zero, as $t$ tends to infinity.

We are now able to state and prove the promised functional central limit theorem:

Theorem 3.3 (Functional central limit theorem). - Let us assume (3.1-ii). Define for each $s>0$ the process $B^{s}:\left(\Omega \times C\left(\mathbb{R}_{+}, \mathbb{R}^{d}\right), \mathscr{A} \otimes \mathscr{F}\right) \rightarrow\left(C\left(\mathbb{R}_{+}, \mathbb{R}^{d}\right), \mathscr{F}\right)$, with

$$
B_{t}^{s}=\frac{X_{s t}-s t v}{\sqrt{s}}, \quad t \geqslant 0 .
$$


Then, under the $\mathrm{P}_{0}$-measure, the $C\left(\mathbb{R}_{+}, \mathbb{R}^{d}\right)$-valued random variable $B_{\text {. }}^{\text {s }}$ converges in law, as $s \rightarrow \infty$, to a d-dimensional Brownian motion B., which has the non-degenerated covariance matrix

$$
\mathbf{K} \stackrel{\text { def }}{=} \frac{\widehat{E}_{0}\left[\left(X_{\tau_{1}}-v \tau_{1}\right)\left(X_{\tau_{1}}-v \tau_{1}\right)^{t} \mid D=\infty\right]}{\widehat{E}_{0}\left[\tau_{1} \mid D=\infty\right]} .
$$

Before proving this theorem, let us recall some classical facts about weak convergence on $C\left(\mathbb{R}_{+}, \mathbb{R}^{d}\right)$, which will be used throughout the proof. (For a detailed treatment, we refer to Chapter 3 in [7], and Section 3.1 in [28].)

On the space $\left(C\left(\mathbb{R}_{+}, \mathbb{R}^{d}\right)\right.$ we define the metric

$$
\rho(Y . ; Z .) \stackrel{\text { def }}{=} \sum_{m=1}^{\infty} \frac{1}{2^{m}} \sup _{0 \leqslant s \leqslant m}\left(\left|Y_{s}-Z_{s}\right| \wedge 1\right) \leqslant 1, \quad Y ., Z . \in C\left(\mathbb{R}_{+}, \mathbb{R}^{d}\right),
$$

which induces the topology of uniform convergence on compact intervals of $\mathbb{R}_{+}$. If on some probability space, say $(\Xi, \mathcal{A}, \mathrm{P}), Y^{n}$. and $Z^{n}$. are sequences of continuous $\mathbb{R}^{d}$-valued stochastic processes, and the laws of the processes $Y^{n}$ converges weakly to some probability measure $Q$ on $C\left(\mathbb{R}_{+}, \mathbb{R}^{d}\right)$, further if $\rho\left(Y_{.}^{n}, Z_{.}^{n}\right)$ converges in probability $\mathrm{P}$ to 0 , then the sequence of laws of the processes $Z^{n}$. converges weakly to $\mathrm{Q}$.

Proof of Theorem 3.3. - It suffices to prove that $B . \stackrel{s \rightarrow \infty}{\longrightarrow} B$. in law under $\widehat{\mathrm{P}}_{0}$, because $X$. has the same distribution under $\widehat{P}_{0}$ and $P_{0}$. The proof is divided in 5 steps. In steps $1-3$, we prove that for integer-valued $s, B \stackrel{s \rightarrow \infty}{\longrightarrow} B$. in law under $\widehat{\mathrm{P}}_{0}$. In step 4 , we generalize this to non-integer $s$. And in the last step, step 5, the non-degeneracy of the covariance matrix $\mathbf{K}$ is proved.

Step 1. Define

$$
\begin{gathered}
Z_{j} \stackrel{\text { def }}{=}\left(X_{\tau_{j+1}}-X_{\tau_{j}}\right)-v\left(\tau_{j+1}-\tau_{j}\right), \quad j \geqslant 1, \\
S_{n} \stackrel{\text { def }}{=} \sum_{j=1}^{n} Z_{j}=X_{\tau_{n+1}}-X_{\tau_{1}}-v\left(\tau_{n+1}-\tau_{1}\right),
\end{gathered}
$$

and let $S_{t}$ be the linear interpolation of $S_{n}$, with the convention $S_{0}=0$.

In view of Theorem 2.5 and the definition of $v$ in (3.7), the random variables $Z_{j}, j \geqslant 1$, are i.i.d., centered under $\widehat{\mathrm{P}}_{0}$, and, thanks to our assumption (3.1-ii) and Lemma 3.1, square integrable.

The Wiener \& Donsker's Invariance Principle, cf. p. 172 in [28], implies that under the $\widehat{\mathrm{P}}_{0}$-measure

$$
\frac{1}{\sqrt{n}} S_{n} \stackrel{n \rightarrow \infty}{\longrightarrow} \widetilde{B} . \quad \text { in law }
$$

where $\widetilde{B}$. is a $d$-dimensional Brownian motion with covariance matrix $\tilde{A}=\widehat{\mathrm{E}}_{0}\left[\tau_{1} \mid D=\right.$ $\infty] \cdot \mathbf{K}$. (The theorem stated in [28] is for the case with covariance matrix equals $\mathbb{1}$. To get our result, we observe that, as we will show below in step 5, the matrix $\tilde{A}$ is positive 
definite, hence $\tilde{A}^{-1 / 2}\left(1 / \sqrt{n} S_{n}\right.$.) converges under $\widehat{\mathrm{P}}_{0}$ in law to a Brownian motion with covariance matrix $\widehat{\mathrm{E}}_{0}\left[\left(\tilde{A}^{-1 / 2} Z_{1}\right)\left(\tilde{A}^{-1 / 2} Z_{1}\right)^{t}\right]=\mathbb{1}$. Thereafter, (3.14) follows.)

Step 2. For each $n \in \mathbb{N}$, define a non-decreasing sequence $j(n) \in \mathbb{N}$ (with the convention $j(0)=0$ ), which tends to infinity $\widehat{P}_{0}$-a.s., such that

$$
\tau_{j(n)} \leqslant n<\tau_{j(n)+1},
$$

and let $j(t)$ be its linear interpolation.

The goal of this step is to show that under $\widehat{\mathrm{P}}_{0}$

$$
\frac{1}{\sqrt{n}} S_{(j(n \cdot)-1)_{+}} \stackrel{n \rightarrow \infty}{\longrightarrow} B . \quad \text { in law }
$$

where $B$. is a $d$-dimensional Brownian motion with the covariance matrix $\mathbf{K}$.

As a result of (3.14), we have $\frac{1}{\sqrt{n}} S_{n \cdot / \hat{E}_{0}\left[\tau_{1} \mid D=\infty\right]} \stackrel{n \rightarrow \infty}{\longrightarrow} B$. in law under $\widehat{\mathrm{P}}_{0}$, so in view of the comments after Theorem 3.3, it suffices to show

$$
\widehat{\mathrm{E}}_{0}\left[\rho\left(\frac{1}{\sqrt{n}} S_{(j(n \cdot)-1)_{+}} ; \frac{1}{\sqrt{n}} S_{\overline{\mathrm{E}_{0}\left[\tau_{1} \mid D=\infty\right]}}\right)\right] \stackrel{n \rightarrow \infty}{\longrightarrow} 0 .
$$

To prove this, we pick $\delta>0$ arbitrarily small, and choose $T \in \mathbb{N}$ large such that $\sum_{m>T} \frac{1}{2^{m}} \leqslant \delta$. Because $\frac{1}{\sqrt{n}} S_{n} \stackrel{n \rightarrow \infty}{\longrightarrow} \widetilde{B}$. in law under $\widehat{\mathrm{P}}_{0}$, the laws of $\frac{1}{\sqrt{n}} S_{n}$. on $\left(C\left(\mathbb{R}_{+}, \mathbb{R}^{d}\right)\right.$ are tight, so there is a compact set $K_{\delta} \subset C\left(\mathbb{R}_{+}, \mathbb{R}^{d}\right)$, for the topology of uniform convergence on compact intervals, such that $\sup _{n} \widehat{\mathrm{P}}_{0}\left[\frac{1}{\sqrt{n}} S_{n} . \notin K_{\delta}\right] \leqslant \delta$, and by the Arzela-Ascoli Theorem, cf. p. 369 in [26], there exists some $\eta(\delta)>0$ such that

$$
\sup _{n} \widehat{\mathrm{P}}_{0}\left[\sup _{\substack{\left|t-t^{\prime}\right| \leqslant \eta \\ t, t^{\prime} \leqslant T}} \frac{1}{\sqrt{n}}\left|S_{n t}-S_{n t^{\prime}}\right| \geqslant \delta\right] \leqslant \delta .
$$

On the other hand, we observe that $|j(t)-j([t])| \leqslant 1, t \in \mathbb{R}_{+}([t]$ denotes the integer part of $t$ ), and

$$
j(m) \leqslant m, \quad \text { for all } m \in \mathbb{N} .
$$

From (3.9), we also see that $j(n)=k(n)$ for all $n \in \mathbb{N}$, hence (3.10) implies $\widehat{\mathrm{P}}_{0}$-a.s. $j(n) / n \stackrel{n \rightarrow \infty}{\longrightarrow} 1 / \widehat{\mathrm{E}}_{0}\left[\tau_{1} \mid D=\infty\right]$. Applying Dini's second lemma, we obtain that

$$
\widehat{\mathrm{P}}_{0} \text {-a.s., for all } U>0, \quad \sup _{0 \leqslant t \leqslant U}\left|\frac{(j(t n)-1)_{+}}{n}-\frac{t}{\widehat{\mathrm{E}}_{0}\left[\tau_{1} \mid D=\infty\right]}\right| \stackrel{n \rightarrow \infty}{\longrightarrow} 0 .
$$

Hence, for $n$ large enough we get

$$
\widehat{\mathrm{P}}_{0}\left[\sup _{0 \leqslant t \leqslant T}\left|\frac{(j(t n)-1)_{+}}{n}-\frac{t}{\widehat{\mathrm{E}}_{0}\left[\tau_{1} \mid D=\infty\right]}\right| \geqslant \eta(\delta)\right] \leqslant \delta .
$$

Coming back to (3.18), we obtain 


$$
\widehat{\mathrm{E}}_{0}\left[\sup _{0 \leqslant t \leqslant T} \frac{1}{\sqrt{n}}\left|S_{(j(t n)-1)_{+}}-S_{t n / \widehat{\mathrm{E}}_{0}\left[\tau_{1} \mid D=\infty\right]}\right| \wedge 1\right] \leqslant 3 \delta,
$$

for sufficiently large $n$. The claim (3.17), and hence (3.16) follow.

Step 3. We show in this step that under $\widehat{P}_{0}$

$$
\frac{1}{\sqrt{n}} B^{n} \stackrel{n \rightarrow \infty}{\longrightarrow} B . \quad \text { in law. }
$$

As stated in the comments after Theorem 3.3, it suffices to show that

$$
\widehat{\mathrm{E}}_{0}\left[\rho\left(B^{n} ; \frac{1}{\sqrt{n}} S_{(j(n \cdot)-1)_{+}}\right)\right] \stackrel{n \rightarrow \infty}{\longrightarrow} 0 .
$$

To this end, choose $T>0$. Then we have

$$
\begin{aligned}
& \sup _{t \leqslant T}\left|B_{t}^{n}-\frac{1}{\sqrt{n}} S_{(j(t n)-1)_{+}}\right| \\
& \quad \leqslant \sup _{t \leqslant T} \frac{1}{\sqrt{n}}\left|S_{(j(t n)-1)_{+}}-S_{(j([t n])-1)_{+}}\right|+\sup _{t \leqslant T}\left|B_{t}^{n}-\frac{1}{\sqrt{n}} S_{(j([t n])-1)_{+}}\right| .
\end{aligned}
$$

Observe that the first term on the right-hand side of (3.22) is bounded from above by

$$
\frac{|v|}{\sqrt{n}} \sup _{0 \leqslant m \leqslant j([T n])}\left(\tau_{m+1}-\tau_{m}\right)+\sup _{0 \leqslant m \leqslant j([T n])} \frac{1}{\sqrt{n}}\left|X_{\tau_{m+1}}-X_{\tau_{m}}\right|,
$$

which, as we will see, converges to 0 in $\widehat{P}_{0}$-probability. Indeed, in view of Theorem 2.5 and (3.19), for any $u>0$ :

$$
\begin{aligned}
& \widehat{\mathrm{P}}_{0}\left[\frac{1}{\sqrt{n}} \sup _{0 \leqslant m \leqslant j([T n])}\left(\tau_{m+1}-\tau_{m}\right)>u\right] \\
& \quad \leqslant \widehat{\mathrm{P}}_{0}\left[\tau_{1}>\sqrt{n} u\right]+[n T] \widehat{\mathrm{P}}_{0}\left[\tau_{1}>\sqrt{n} u \mid D=\infty\right] \\
& \quad \leqslant \widehat{\mathrm{P}}_{0}\left[\tau_{1}>\sqrt{n} u\right]+\frac{n T}{n u^{2}} \widehat{\mathrm{E}}_{0}\left[\tau_{1}^{2}, \tau_{1}>\sqrt{n} u \mid D=\infty\right] \stackrel{n \rightarrow \infty}{\longrightarrow} 0,
\end{aligned}
$$

by assumption (3.1-ii). Similar result holds for the second term in (3.23), by (3.2-ii) we have:

$$
\begin{aligned}
\widehat{\mathrm{P}}_{0} & {\left[\frac{1}{\sqrt{n}} \sup _{0 \leqslant m \leqslant j([T n])}\left|X_{\tau_{m+1}}-X_{\tau_{m}}\right|>u\right] } \\
& \leqslant \widehat{\mathrm{P}}_{0}\left[\left|X_{\tau_{1}}\right|>\sqrt{n} u\right]+\frac{n T}{n u^{2}} \widehat{\mathrm{E}}_{0}\left[\left|X_{\tau_{1}}\right|^{2},\left|X_{\tau_{1}}\right|>\sqrt{n} u \mid D=\infty\right] \stackrel{n \rightarrow \infty}{\longrightarrow} 0 .
\end{aligned}
$$

Let us now consider the second term on the right-hand side of (3.22). We claim that it also converges in $\widehat{P}_{0}$-probability to 0 . To show this, we start with the easy fact that $\widehat{\mathrm{P}}_{0}$-a.s., the second term on r.h.s. of (3.22) is smaller than

$$
\sup _{t \leqslant T} \frac{1}{\sqrt{n}}\left\{\int_{\tau_{j([n t])}}^{n t}\left(|v|+\left|b\left(X_{s}, \omega\right)\right|\right) d s+\int_{0}^{\tau_{1}}\left(|v|+\left|b\left(X_{s}, \omega\right)\right|\right) d s\right\}
$$




$$
+\sup _{t \leqslant T} \frac{1}{\sqrt{n}}\left\{\left|Y_{n t}-Y_{\tau_{j([n t])}}\right|+\left|Y_{\tau_{1}}\right|\right\}
$$

with $Y_{t}$ defined in (3.4). The first term in (3.24) is bounded from above by

$$
\frac{\bar{b}+|v|}{\sqrt{n}} \sup _{t \leqslant T}\left(n t-\tau_{j([n t])}+\tau_{1}\right) \leqslant \frac{2(\bar{b}+|v|)}{\sqrt{n}}\left(\sup _{0 \leqslant m \leqslant j([n T])}\left(\tau_{m+1}-\tau_{m}\right)\right),
$$

which converges to 0 in $\widehat{P}_{0}$-probability, as shown above.

The last term in (3.24) is smaller than $\frac{1}{\sqrt{n}}\left|Y_{\tau_{1}}\right|+\frac{1}{\sqrt{n}} \sup _{t \leqslant T}\left|Y_{n t}-Y_{\tau_{j([n t])}}\right|$, which, we claim, converges also to 0 in $\widehat{\mathrm{P}}_{0}$-probability. Indeed, for all $u>0$ :

$$
\begin{aligned}
& \widehat{\mathrm{P}}_{0}\left[\sup _{t \leqslant T}\left|Y_{n t}-Y_{\tau_{j([n t])}}\right|>\sqrt{n} u\right] \\
& \leqslant \widehat{\mathrm{P}}_{0}\left[\sup _{t \leqslant T}\left|Y_{n t}-Y_{\tau_{j([n t])}}\right|>\sqrt{n} u ; \sup _{0 \leqslant m \leqslant j([n T])}\left(\tau_{m+1}-\tau_{m}\right) \leqslant \sqrt{n}\right] \\
& +\widehat{\mathrm{P}}_{0}\left[\sup _{0 \leqslant m \leqslant j([n T])}\left|\tau_{m+1}-\tau_{m}\right|>\sqrt{n}\right] \text {. }
\end{aligned}
$$

We know already from above that the second term on the r.h.s. of (3.25) converges to 0 , as $n \rightarrow \infty$. For the first term on the r.h.s. in (3.25), we observe that it can be further estimated from above by

$$
\begin{aligned}
& \widehat{\mathrm{P}}_{0}\left[\sup _{m \leqslant[n T]} \sup _{0 \leqslant s \leqslant \sqrt{n}}\left|Y_{m+s}-Y_{m}\right|>\sqrt{n} u\right] \\
& \leqslant \sum_{m=0}^{[n T]} \widehat{\mathrm{P}}_{0}\left[\sup _{0 \leqslant s \leqslant \sqrt{n}}\left|Y_{m+s}-Y_{m}\right|>\sqrt{n} u\right] .
\end{aligned}
$$

Applying the Bernstein's inequality, cf. pp. 153-154 in [25], we obtain that for any $m \in \mathbb{N}$ :

$$
\mathrm{P}_{0}^{\omega}\left[\sup _{s \leqslant \sqrt{n}}\left|Y_{m+s}-Y_{m}\right|>\sqrt{n} u\right] \leqslant 2 d \mathrm{e}^{-u^{2} \sqrt{n} /(2 v)},
$$

thus the right-hand side of (3.26) tends to 0. This completes the proof of (3.20).

Step 4. In this step we study $B_{\text {. }}^{s}$ for $s \in \mathbb{R}_{+}$tending to infinity, and extends (3.20).

The proof is very similar the one given in step 2. We consider $s_{n} \rightarrow \infty$. For $\delta>0$ arbitrarily small, we define $T \in \mathbb{N}$ such that $\sum_{m>T} \frac{1}{2^{m}} \leqslant \delta$.

From (3.21) we know that under $\widehat{\mathrm{P}}_{0}$, with $B$. as in (3.16),

$$
\frac{X_{\left[s_{n}\right] \cdot}-v\left[s_{n}\right]}{\sqrt{\left[s_{n}\right]}}, \quad \text { and hence } \quad \frac{X_{\left[s_{n}\right] \cdot}-v\left[s_{n}\right]}{\sqrt{s_{n}}},
$$

converges in law to $B$., as $n \rightarrow \infty$.

Therefore, the laws of $\frac{1}{\sqrt{s_{n}}}\left(X_{\left[s_{n}\right]}-v\left[s_{n}\right] \cdot\right)$ are tight, and for any $T>0$ and $\delta>0$, one can find $\eta(\delta)>0$ such that: 


$$
\sup _{n} \widehat{\mathrm{P}}_{0}\left[\sup _{\substack{\mid t-t^{\prime} \leqslant \uparrow \\ t, t^{\prime} \leqslant T}} \frac{\left(X_{\left[s_{n}\right] t}-v\left[s_{n}\right] t\right)-\left(X_{\left[s_{n}\right] t^{\prime}}-v\left[s_{n}\right] t^{\prime}\right)}{\sqrt{s_{n}}} \geqslant \delta\right] \leqslant \delta .
$$

Since $\sup _{t \leqslant T}\left|t-\left(s_{n} /\left[s_{n}\right]\right) t\right| \stackrel{n \rightarrow \infty}{\longrightarrow} 0$, we obtain that for large $n$

$$
\widehat{\mathrm{P}}_{0}\left[\sup _{0 \leqslant t \leqslant T} \frac{1}{\sqrt{s_{n}}}\left|\left(X_{\left[s_{n}\right] t}-v\left[s_{n}\right] t\right)-\left(X_{s_{n} t}-v s_{n} t\right)\right| \geqslant \delta\right] \leqslant \delta,
$$

and from (3.27) we deduce our claim.

Step 5. In this final step we will prove the non-degeneracy of the covariance matrix $\mathbf{K}$.

First, we let $H \stackrel{\text { def }}{=}\left\{z \in \mathbb{R}^{d}: 14 R<z \cdot \ell<15 R\right\}$ be a strip in $\mathbb{R}^{d}$. We claim that for any $n \geqslant 3$ and $x \in H$,

$$
\begin{aligned}
& \widehat{\mathrm{P}}_{0}\left[X_{\tau_{1}} \in B_{R}(x) ; n=S_{1}=\tau_{1} ; D=\infty\right] \\
& \quad=\widehat{\mathrm{P}}_{0}\left[X_{n} \in B_{R}(x) ; n=S_{1}<D\right] \cdot \mathrm{P}_{0}[D=\infty]>0 .
\end{aligned}
$$

To show this, we prove in the first step that for any $x \in H$

$$
\widehat{\mathrm{P}}_{0}\left[X_{n} \in B_{R}(x) ; n=S_{1}<D\right]>0 .
$$

To see this, we observe that for all $\omega \in \Omega, x \in H$, with $\widetilde{B} \stackrel{\text { def }}{=}\left\{z \in \mathbb{R}^{d}:\left|B^{z} \cap B_{R}(x)\right| \geqslant\right.$ $\left.\left|B_{R}\right| / 2\right\}$ (recall (2.1)), we have (see (2.13), (2.14) and Theorem 2.1):

$$
\begin{gathered}
\widehat{\mathrm{P}}_{0}^{\omega}\left[X_{n} \in B_{R}(x) ; n=S_{1}<D\right] \geqslant \frac{1}{2} \widehat{\mathrm{P}}_{0}^{\omega}\left[X_{n-1} \in \widetilde{B} ; N_{1}=n-1 ; \widetilde{T}_{-R}>n-1\right] \\
\geqslant \frac{\varepsilon}{2} \mathrm{P}_{0}^{\omega}\left[X_{n-1} \in \widetilde{B} ; \widetilde{N}_{1}(3 R)=\left[V_{1}(3 R)\right]=n-1 ; \widetilde{T}_{-R}>n-1\right] .
\end{gathered}
$$

Because the path in Fig. 5 belongs to the event on the right-hand side of (3.30), with the Support Theorem of Stroock-Varadhan, cf. p. 25 in [2], the right-hand side in (3.30) is positive, for all $\omega \in \Omega$. This proves (3.29).

To finish the proof of (3.28), we only need to prove the first equality in (3.28). To do this, we proceed as in the proof of Theorem 2.4:

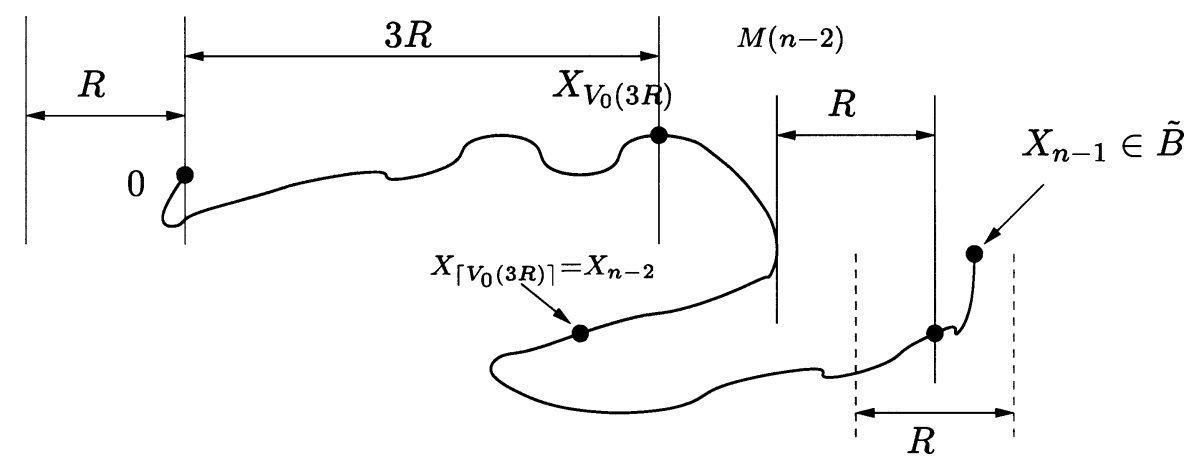

Fig. 5. 


$$
\begin{aligned}
\widehat{\mathrm{P}}_{0} & {\left[X_{\tau_{1}} \in B_{R}(x) ; n=S_{1}=\tau_{1} ; D=\infty\right] } \\
& =\widehat{\mathrm{P}}_{0}\left[X_{n} \in B_{R}(x) ; n=S_{1}<D ; D \circ \theta_{n}=\infty\right] \\
& =\mathbb{E}\left\{\widehat{\mathrm{P}}_{0}^{\omega}\left[X_{n-1} \in \widehat{B} ; \lambda_{n-1}=1 ; \Gamma ; X_{1} \circ \theta_{n-1} \in B_{R}(x) ; D \circ \theta_{n}=\infty\right]\right\},
\end{aligned}
$$

with $\widehat{B} \stackrel{\text { def }}{=}\left\{z \in \mathbb{R}^{d}: B^{z} \cap B_{R}(x) \neq 0\right\}$ and some $\Gamma \in \mathscr{F}_{n-1} \otimes \mathscr{S}_{n-2}$. By the Markov property, cf. Corollary 2.2, and similar calculations as in the proof of Theorem 2.4, see p. 11, the last expression equals

$$
\frac{1}{\left|B_{R}\right|} \int d y \mathbb{E}\left\{\widehat{\mathrm{P}}_{0}^{\omega}[V] \cdot \widehat{\mathrm{P}}_{y}^{\omega}[D=\infty]\right\}=\frac{1}{\left|B_{R}\right|}\left(\int d y \widehat{\mathrm{P}}_{0}[V]\right) \cdot \mathrm{P}_{0}[D=\infty],
$$

with $V \stackrel{\text { def }}{=}\left\{X_{n-1} \in \widehat{B} ; \Gamma ; \lambda_{n-1}=1 ; y \in B^{X_{n-1}} \cap B_{R}(x)\right\}$, where, as in the proof of Theorem 2.4, we have used that $\widehat{\mathrm{P}}_{0}^{\omega}[V]$ and $\mathrm{P}_{y}^{\omega}[D=\infty]$ are $\mathbb{P}$-independent, and the $\mathbb{P}$-measure is translation invariant. On the other hand, we observe that by the identical calculation $\widehat{\mathrm{P}}_{0}\left[X_{n} \in B_{R}(x) ; n=S_{1}<D\right]=\int d y \frac{1}{\left|B_{R}\right|} \widehat{\mathrm{E}}_{0}[V]$ holds, the first equality in (3.28) follows immediately.

With the help of (3.28) we can now prove the non-degeneracy of the covariance matrix $\mathbf{K}$. Clearly, for any $w \in \mathbb{R}^{d}, w^{t} \mathbf{K} w \geqslant 0$, i.e. $\mathbf{K}$ is positive semi-definite. We prove the non-degeneracy by contradiction. If $w^{t} \mathbf{K} w=0$ for some unit vector $w \in \mathbb{R}^{d}$, then $\widehat{\mathrm{P}}_{0}\left[w \cdot\left(X_{\tau_{1}}-\tau_{1} v\right)=0 \mid D=\infty\right]=1$.

Combine this with (3.28), we obtain that for any given $x \in H$, and for all $n \geqslant 3$ : $\widehat{\mathrm{P}}_{0}\left[w \cdot x-R \leqslant n(w \cdot v) \leqslant w \cdot x+R ; \tau_{1}=n \mid D=\infty\right]>0$, which implies $w \cdot v=0$. Coming back to the above inequality, we see that $|w \cdot x| \leqslant R$ for $x \in H$, by taking limits of points in $H$, we obtain that $w \cdot z=0$, for all $z$ such that $z \cdot \ell=0$. Since $v \cdot \ell>0$, it follows that $w=0$. This, combined with $\widehat{\mathrm{E}}_{0}\left[\tau_{1} \mid D=\infty\right]<\infty$, proves the non-degeneracy of the matrix $\mathbf{K}$, and hence finish the proof of Theorem 3.3.

\section{Application to an anisotropic gradient-type diffusion}

In this section we will apply the results from the previous sections to a class of anisotropic diffusion processes in a random medium, which is reversible when the environment is fixed. The class under consideration is a specialization of (1.7) with $\sigma=\mathbb{1}$ and $b(x, \omega)=\nabla V(x, \omega)$, where for each $\omega \in \Omega, V(\cdot, \omega) \in C^{1}\left(\mathbb{R}^{d}, \mathbb{R}\right)$ has bounded and Lipschitz-continuous derivatives; in addition we assume that for some $\ell \in S^{d-1}, A, B>0$ and $\lambda>0$,

$$
A \mathrm{e}^{2 \lambda \ell \cdot x} \leqslant \mathrm{e}^{2 V(x, \omega)} \leqslant B \mathrm{e}^{2 \lambda \ell \cdot x}, \quad \text { for } x \in \mathbb{R}^{d}, \omega \in \Omega .
$$

We will prove the existence of an effective, non-vanishing velocity, and a functional central limit theorem in Theorem 4.11.

Let us mention that in this section $c, \tilde{c}, \hat{c}$ and $C$ always denote some positive constants, which do not depend on $x \in \mathbb{R}^{d}$ and $\omega \in \Omega$. They need not to be the same in each occurrence. 


\subsection{Key estimates}

We will now derive estimates on the exit distribution and exit time of the diffusion process from a large cylinder with axis parallel to $\ell$, cf. Propositions 4.1 and 4.2. We will then derive the transience of the process in direction $\ell$, cf. Corollary 4.6.

Let us introduce

$$
m_{\omega}(d x) \stackrel{\text { def }}{=} \exp \{2 V(x, \omega)\} d x, \quad m(d x) \stackrel{\text { def }}{=} \exp \{2 \lambda \ell \cdot x\} d x,
$$

and the corresponding scalar product $(\cdot ; \cdot)_{m_{\omega}}$ on $L^{2}\left(m_{\omega}\right)$, respectively $(\cdot ; \cdot)_{m}$ on $L^{2}(m)$. Observe that due to (4.1), the norms $\|\cdot\|_{L^{2}\left(m_{\omega}\right)}$ and $\|\cdot\|_{L^{2}(m)}$ are equivalent, hence $L^{2}\left(m_{\omega}\right)=L^{2}(m)$ for all $\omega \in \Omega$.

Further, let us denote by $\left(P_{\omega}^{t}\right)_{t \geqslant 0}$ the semi-group corresponding to the solution of this stochastic differential equation, that is, $\left(P_{\omega}^{t} f\right)(x)=\mathrm{E}_{x}^{\omega}\left[f\left(X_{t}\right)\right]$ for $f$ bounded and Borel-measurable. Observe that for each $\omega \in \Omega$ the differential operator

$$
L_{\omega}=\frac{1}{2} \Delta+\nabla V(x, \omega) \cdot \nabla
$$

is the generator of the semi-group $\left(P_{\omega}^{t}\right)_{t \geqslant 0}$, cf. page 251 in [6], One can easily check that $\left(f ; L_{\omega} g\right)_{m_{\omega}}=\left(g ; L_{\omega} f\right)_{m_{\omega}}$ for $f, g \in C_{c}^{\infty}\left(\mathbb{R}^{d}, \mathbb{R}\right)$. From (2.3) in [10] we observe that $m_{\omega}(d x)$ is the reversible measure to $P_{\omega}^{t}$, i.e. $\left(f ; P_{\omega}^{t} g\right)_{m_{\omega}}=\left(g ; P_{\omega}^{t} f\right)_{m_{\omega}}$, for $f, g \in$ $L^{1}\left(m_{\omega}\right)$ and bounded (the operator $L_{\omega}$ has the form of (3.4) in [10], therefore the assumption for (2.3) in [10] is fulfilled).

Let us now introduce the Dirichlet form $\mathscr{E}_{m_{\omega}}$ corresponding to the operator $L_{\omega}$, or the semi-group $P_{\omega}^{t}$,

$$
\mathscr{E}_{m_{\omega}}(f, g) \stackrel{\text { def }}{=} \lim _{t \downarrow 0} \frac{1}{t}\left(\left(1-P_{\omega}^{t}\right) f ; g\right)_{m_{\omega}},
$$

with its definition domain $\mathscr{D}_{m_{\omega}} \stackrel{\text { def }}{=}\left\{f \in L^{2}\left(m_{\omega}\right)\right.$ : $\left.\lim _{t \downarrow 0} \frac{1}{t}\left(\left(1-P_{\omega}^{t}\right) f ; f\right)_{m_{\omega}}<\infty\right\}$. It follows from Remark (2.12) and the proof of Theorem (2.3) in [10] that $C_{c}^{\infty}\left(\mathbb{R}^{d}, \mathbb{R}\right)$ is a core of $\mathscr{E}_{m_{\omega}}$. Further, from (4.1), we have

$$
\left\{\begin{array}{l}
\mathscr{D}_{m_{\omega}}=\mathscr{D}=\left\{f \in L^{2}(m): \frac{\partial}{\partial x_{i}} f \in L^{2}(m), i=1, \ldots, d\right\}, \\
\mathscr{E}_{m_{\omega}}(f, g)=\frac{1}{2} \sum_{i=1}^{d}\left(\frac{\partial}{\partial x_{i}} f ; \frac{\partial}{\partial x_{i}} g\right)_{m_{\omega}}, \quad f, g \in \mathscr{D}, \\
A \mathscr{E}_{m}(f, f) \leqslant \mathscr{E}_{m_{\omega}}(f, f) \leqslant B \mathscr{E}_{m}(f, f), \quad f \in \mathscr{D},
\end{array}\right.
$$

with $\mathscr{E}_{m}(f, g)=\frac{1}{2} \sum_{i=1}^{d}\left(\frac{\partial}{\partial x_{i}} f ; \frac{\partial}{\partial x_{i}} g\right)_{m}$.

For each $\omega \in \Omega$ and open subset $U$ of $\mathbb{R}^{d}$, we introduce the bottom of the Dirichlet spectrum of operator $-L_{\omega}$ in $U$ :

$$
\Lambda_{\omega}(U)=\inf \left\{\frac{\mathscr{E}_{m_{\omega}}(f, f)}{(f ; f)_{m_{\omega}}}: f \in C_{c}^{\infty}(U), f \neq 0\right\} \geqslant 0 .
$$


PROPOSITION 4.1.-

$$
\inf _{U, \omega \in \Omega} \Lambda_{\omega}(U)>0
$$

where $U$ varies over the collection of non-empty open subsets of $\mathbb{R}^{d}$. The bounded selfadjoint operator $P_{\omega, U}^{t}$ on $L^{2}\left(m_{\omega}\right)$, which is defined by $\left(P_{\omega, U}^{t} f\right)(x) \stackrel{\text { def }}{=} \mathrm{E}_{x}^{\omega}\left[f\left(X_{t}\right), T_{U}>t\right]$, for $t>0$ and $f \in L^{2}\left(m_{\omega}\right)$, satisfies

$$
\sup _{\omega, U}\left\|P_{\omega, U}^{t}\right\|_{m_{\omega}} \leqslant \exp \left\{-\frac{\gamma t}{\lambda}\right\}, \quad t>0,
$$

for some $\gamma>0$, with $\|\cdot\|_{m_{\omega}}$ denoting the operator norm in $L^{2}\left(m_{\omega}\right)$.

Proof. - Observe that because of (4.1) the inequality $\frac{1}{B}(f ; f)_{m_{\omega}} \leqslant(f ; f)_{m}$ holds for all $f \in L^{2}(m)=L^{2}\left(m_{\omega}\right)$; and similarly $\frac{1}{A} \mathscr{E}_{m_{\omega}}(f, f) \geqslant \mathscr{E}_{m}(f, f)$ holds for all $f \in$ $C_{c}^{\infty}(U)$. Therefore, for $U$ open subset of $\mathbb{R}^{d}, \Lambda_{\omega}(U) \geqslant \frac{A}{B} \Lambda(U)$, for all $\omega \in \Omega$, where $\Lambda(U)$ is defined, analogously to $\Lambda_{\omega}(U)$ in (4.5), with $\mathscr{E}_{m}$ instead of $\mathscr{E}_{m_{\omega}}$ and with $(\cdot ; \cdot)_{m}$ instead of $(\cdot ; \cdot)_{m_{\omega}}$.

It thus suffices to find a lower bound for $\inf _{U} \Lambda(U)$. Further, because $\Lambda\left(\mathbb{R}^{d}\right)=$ $\inf _{U \neq \emptyset} \Lambda(U)$ and (4.5) also holds for $\Lambda(U)$, we can assume that $U$ is open and bounded.

Observe that the measure $m(d x)=\mathrm{e}^{2 \lambda \ell \cdot x} d x$ is (up to a multiplication factor) the reversible measure for Brownian motion with constant drift $\lambda \ell$, and $\mathscr{E}_{m}$ is just the corresponding Dirichlet form. Let us denote the canonical law of this diffusion process starting in $x$ by $\mathrm{Q}_{x}$ and its expectation value by $\mathrm{E}_{x}^{\mathrm{Q}}$. Then $\exp \left\{-\delta \ell \cdot X_{t}+\alpha t\right\}$ is a $\mathrm{Q}_{x}$-martingale, provided $\alpha=\delta \lambda-\delta^{2} / 2$. Choosing $\delta>0$ small enough, we can make $\alpha>0$. The stopping theorem implies that for any bounded open set $U \subset \mathbb{R}^{d}$ containing $x, \mathrm{E}_{x}^{\mathrm{Q}}\left[\exp \left\{-\delta \ell \cdot\left(X_{T_{U}}-x\right)+\alpha T_{U}\right\}\right]=1$. With $\rho \stackrel{\text { def }}{=} \sup \left\{\left|\ell \cdot\left(z-z^{\prime}\right)\right|: z, z^{\prime} \in U\right\}$, we have $-\delta \ell \cdot\left(X_{T_{U}}-x\right) \geqslant-\delta \rho$, hence $\sup _{x \in U} \mathrm{E}_{x}^{\mathrm{Q}}\left[\exp \left\{\alpha T_{U}\right\}\right] \leqslant \mathrm{e}^{\delta \rho}$.

Now, let us introduce the bounded self-adjoint operator $\mathrm{Q}_{U}^{t}$ on $L^{2}(m)$, which is defined by $\left(\mathrm{Q}_{U}^{t} f\right)(x) \stackrel{\text { def }}{=} \mathrm{E}_{x}^{\mathrm{Q}}\left[f\left(X_{t}\right), T_{U}>t\right]$, with $t>0$ and $f \in L^{2}(m)$. We claim that for all $t>0$ :

$$
\sup _{\substack{U \text { open } \\ U \neq \emptyset}}\left\|\mathrm{Q}_{U}^{t}\right\|_{m} \leqslant \mathrm{e}^{-\alpha t / 2}
$$

with $\|\cdot\|_{m}$ denoting the operator norm in $L^{2}(m)$. To show this, we observe that for $f \in L^{2}(m)$ :

$$
\begin{aligned}
\left\|\mathrm{Q}_{U}^{t} f\right\|_{L^{2}(m)}^{2} & =\int_{U} m(d x)\left(\mathrm{Q}_{U}^{t} f\right)^{2}(x) \stackrel{\text { Jensen }}{\leqslant}\left(1_{U} ; \mathrm{Q}_{U}^{t} f^{2}\right)_{m} \\
& =\left(\mathrm{Q}_{U}^{t} 1_{U} ; f^{2}\right)_{m}=\int m(d y) \mathrm{Q}_{y}\left[T_{U}>t\right] f^{2}(y) \leqslant \mathrm{e}^{-\alpha t} \mathrm{e}^{\delta \rho}\|f\|_{L^{2}(m)}^{2},
\end{aligned}
$$

where Chebychev's inequality $\mathrm{Q}_{y}\left[T_{U}>t\right] \leqslant \mathrm{E}_{y}^{\mathrm{Q}}\left[\mathrm{e}^{\alpha\left(T_{U}-t\right)}\right] \leqslant \mathrm{e}^{-\alpha t+\delta \rho}$ is used in the last step. Hence, $\left\|\mathrm{Q}_{U}^{n t}\right\|_{m}^{2} \leqslant \mathrm{e}^{-\alpha n t+\delta \rho}, n \in \mathbb{N}$. Taking the $n$th root, it follows from Theorem VI.6 on p. 192 in [24], that $\left\|\mathrm{Q}_{U}^{t}\right\|_{m} \leqslant \mathrm{e}^{-\alpha t / 2}$, and our claim follows. This 
implies that $\Lambda(U) \geqslant \frac{\alpha}{2}>0$, and (4.6) follows. Finally, (4.7) is just an easy consequence of (4.6), cf. Theorem 4.4.2 in [9].

Let $U(L)$ now be a cylinder centered at $x$ with height $4 L$ in the direction $\ell$ and radius $4 L^{2}>0$ in the directions normal to $\ell$, that is,

$$
U(L) \stackrel{\text { def }}{=}\left\{z \in \mathbb{R}^{d}:|(z-x) \cdot \ell|<2 L ;|(z-x) \cdot e|<4 L^{2}, \forall e \perp \ell,|e|=1\right\} .
$$

PROPOSITION 4.2. - There exist two constants $c_{1}>0$ and $\tilde{c}_{1}>0$ such that for all $L>0$

$$
\sup _{x, \omega} \mathrm{P}_{x}^{\omega}\left[T_{U(L)} \geqslant \frac{4}{\gamma} L\right] \leqslant \tilde{c}_{1} \mathrm{e}^{-c_{1} L} .
$$

Proof. - Observe that for $t \geqslant 1$,

$$
\mathrm{P}_{x}^{\omega}\left[T_{U(L)}>t\right] \leqslant \mathrm{P}_{x}^{\omega}\left[X_{1} \in B_{L}(x), T_{U(L)} \circ \theta_{1}>t-1\right]+\mathrm{P}_{x}^{\omega}\left[X_{1} \notin B_{L}(x)\right] .
$$

By (A.5), there exist constants $\tilde{c}>0$ and $c>0$ such that the second term on the righthand side above is smaller than $\tilde{c} \mathrm{e}^{-c L^{2}}$ for all $x \in \mathbb{R}^{d}$ and $\omega \in \Omega$, hence it suffices to study the first term in the above expression.

By the Markov property, the first term above is

$$
\begin{aligned}
\mathrm{P}_{x}^{\omega} & {\left[X_{1} \in B_{L}(x), T_{U(L)} \circ \theta_{1}>t-1\right]=\mathrm{E}_{x}^{\omega}\left[X_{1} \in B_{L}(x), \mathrm{P}_{X_{1}}^{\omega}\left[T_{U}>t-1\right]\right] } \\
& =\left(1_{B_{L}(x)}(\cdot) p_{\omega}(1, x, \cdot) \mathrm{e}^{-2 V(\cdot, \omega)} ;\left(P_{\omega, U}^{t-1} 1_{U}\right)(\cdot)\right)_{m_{\omega}} \\
& \leqslant\left\|1_{B_{L}(x)}(\cdot) p_{\omega}(1, x, \cdot) \mathrm{e}^{-2 V(\cdot, \omega)}\right\|_{L^{2}\left(m_{\omega}\right)} \times\left\|P_{\omega, U}^{t-1}\right\|_{m_{\omega}} \times\left\|1_{U}\right\|_{L^{2}\left(m_{\omega}\right)} .
\end{aligned}
$$

Because there exists a constant $c>0$ such that $p_{\omega}(1, x, y) \leqslant c$ for all $\omega \in \Omega, x \in \mathbb{R}^{d}$ and $y \in B_{L}(x)$, cf. (A.9), we obtain for the first term on the rightmost side in the above expression that

$$
\left\|1_{B_{L}(x)} p_{\omega}(1, x, \cdot) \mathrm{e}^{-2 V}\right\|_{m_{\omega}}^{2} \leqslant c^{2} \int d y 1_{B_{L}(x)}(y) \mathrm{e}^{-2 V(y, \omega)} \leqslant \tilde{c} L^{d} \mathrm{e}^{-2 \lambda \ell \cdot x} \mathrm{e}^{2 \lambda L},
$$

for some $\tilde{c}>0$, where we used (4.1) in the last step. Similarly, we can estimate $\left\|1_{U}\right\|_{m_{\omega}}$ by:

$$
\left\|1_{U}\right\|_{m_{\omega}}^{2}=\int d y 1_{U}(y) \mathrm{e}^{2 V(y, \omega)} \leqslant B \int d y 1_{U}(y) \mathrm{e}^{2 \lambda \ell \cdot y} \leqslant c L^{2 d-1} \mathrm{e}^{2 \lambda \ell \cdot x} \mathrm{e}^{4 \lambda L} .
$$

Putting them with (4.7) together, we obtain for $t \geqslant \frac{4 L}{\gamma} \vee 1$ that

$$
\mathrm{P}_{x}^{\omega}\left[T_{U}>t\right] \leqslant \tilde{c} L^{c(d)} \mathrm{e}^{3 \lambda L} \mathrm{e}^{-\gamma(t-1)} \leqslant \tilde{c} \mathrm{e}^{-c L},
$$

for all $x \in \mathbb{R}^{d}$ and $\omega \in \Omega$. Therefore, we can find $\tilde{c}_{1}>0$ and $c_{1}>0$ such that $\mathrm{P}_{x}^{\omega}\left[T_{U(L)} \geqslant\right.$ $\left.\frac{4}{\gamma} L\right] \leqslant \tilde{c}_{1} \mathrm{e}^{-c_{1} L}$. 
Let us divide the boundary of $U(L)$, cf. (4.8), into $\partial U(L)=\partial_{+} U(L) \cup \partial_{-} U(L) \cup$ $\partial_{0} U(L)$, with

$$
\left\{\begin{array}{l}
\partial_{+} U(L) \stackrel{\text { def }}{=}\{z \in \partial U(L): \ell \cdot(z-x) \geqslant 2 L\}, \\
\partial_{-} U(L) \stackrel{\text { def }}{=}\{z \in \partial U(L): \ell \cdot(z-x) \leqslant-2 L\}, \\
\partial_{0} U(L) \stackrel{\text { def }}{=} \partial U(L) \backslash\left(\partial_{+} U(L) \cup \partial_{-} U(L)\right) .
\end{array}\right.
$$

The following estimate will play an important role:

PROPOSITION 4.3. - There exist two constants $c_{2}>0$ and $\tilde{c}_{2}>0$ such that for all $L>0$ :

$$
\sup _{x, \omega} \mathrm{P}_{x}^{\omega}\left[T_{U(L)}<\frac{4 L}{\gamma} ; X_{T_{U(L)}} \notin \partial_{+} U\right] \leqslant \tilde{c}_{2} \mathrm{e}^{-c_{2} L}
$$

Proof. - Without loss of generality let us assume $L>\gamma / 4$. Observe that, with $I_{n} \stackrel{\text { def }}{=}$ $[n, n+1), n \geqslant 0$, we have

$$
\mathrm{P}_{x}^{\omega}\left[T_{U}<\frac{4 L}{\gamma} ; X_{T_{U}} \notin \partial_{+} U\right] \leqslant \mathrm{P}_{x}^{\omega}\left[T_{U} \in I_{0}\right]+\sum_{n=1}^{\lceil 4 L / \gamma\rceil-1} \mathrm{P}_{x}^{\omega}\left[T_{U} \in I_{n}, X_{T_{U}} \notin \partial_{+} U\right] .
$$

Also observe that in the above expression, because of (A.5), we have for the the first term on the right-hand side

$$
\mathrm{P}_{x}^{\omega}\left[T_{U} \in I_{0}\right] \leqslant \mathrm{P}_{x}^{\omega}\left[\sup _{s \leqslant 1}\left|X_{s}-X_{0}\right|>2 L\right] \leqslant \tilde{c} \mathrm{e}^{-c L^{2}}, \quad \text { for all } x \in \mathbb{R}^{d}, \omega \in \Omega .
$$

For the terms in the sum, we notice that for $n \geqslant 1$ :

$$
\begin{aligned}
& \mathrm{P}_{x}^{\omega}\left[T_{U} \in I_{n}, X_{T_{U}} \notin \partial_{+} U\right] \\
& \quad \leqslant \mathrm{P}_{x}^{\omega}\left[X_{1} \in B_{L / 2}(x), T_{U} \in I_{n}, X_{T_{U}} \notin \partial_{+} U\right]+\mathrm{P}_{x}^{\omega}\left[X_{1} \notin B_{L / 2}(x)\right],
\end{aligned}
$$

and $\mathrm{P}_{x}^{\omega}\left[X_{1} \notin B_{L / 2}(x)\right] \leqslant \mathrm{P}_{x}^{\omega}\left[\sup _{s \leqslant 1}\left|X_{s}-X_{0}\right|>L / 2\right] \stackrel{(\mathrm{A} .5)}{\leqslant} \tilde{c} \mathrm{e}^{-c L^{2}}$. Hence, we only need to prove that $\sum_{1 \leqslant n<(4 L / \gamma)} \mathrm{P}_{x}^{\omega}\left[X_{1} \in B_{L / 2}(x), T_{U} \in I_{n}, X_{T_{U}} \notin \partial_{+} U\right] \leqslant \tilde{c} \mathrm{e}^{-c L}$. To this end, we notice that

$$
\begin{aligned}
\mathrm{P}_{x}^{\omega}[ & \left.X_{1} \in B_{L / 2}(x), T_{U} \in I_{n}, X_{T_{U}} \notin \partial_{+} U\right] \\
\leqslant & \mathrm{P}_{x}^{\omega}\left[X_{1} \in B_{L / 2}(x), T_{U} \in I_{n}, X_{n} \in U_{0} \cup U_{-}\right] \\
& +\mathrm{P}_{x}^{\omega}\left[T_{U} \in I_{n}, \sup _{s \leqslant 1}\left|X_{s}-X_{0}\right| \circ \theta_{n}>\frac{L}{2}\right],
\end{aligned}
$$

with $U_{0}(L) \stackrel{\text { def }}{=}\left\{z \in \mathbb{R}^{d}: \exists y \in \partial_{0} U(L),|y-z|<L / 2\right\}$ and $U_{-}(L) \stackrel{\text { def }}{=}\left\{z \in \mathbb{R}^{d}: \exists y \in\right.$ $\left.\partial_{-} U(L),|y-z|<L / 2\right\}$. We see with (A.5) that the expression above is

$$
\leqslant \mathrm{P}_{x}^{\omega}\left[X_{1} \in B_{L / 2}(x), T_{U} \in I_{n}, X_{n} \in U_{0} \cup U_{-}\right]+\tilde{c} \mathrm{e}^{-c L^{2}} .
$$


Thus, it suffices to show that $\sum_{1 \leqslant n<(4 L / \gamma)} \mathrm{P}_{x}^{\omega}\left[X_{1} \in B_{L / 2}(x), X_{n} \in U_{0} \cup U_{-}\right] \leqslant \tilde{c} \mathrm{e}^{-c L}$. To prove this, we observe that with for $U_{j}=U_{0}$ or $U_{j}=U_{-}$, it follows from the Markov property and $p_{\omega}(1, x, y) \leqslant c$, cf. (A.9), that

$$
\begin{aligned}
\mathrm{P}_{x}^{\omega}\left[X_{1} \in B_{L / 2}(x), X_{n} \in U_{j}\right] & =\int_{B_{L / 2}(x)} d z p_{\omega}(1, x, z)\left(P_{\omega}^{n-1} 1_{U_{j}}\right)(z) \\
& \leqslant c \mathrm{e}^{-2 \lambda \ell \cdot x} \mathrm{e}^{\lambda L}\left(P_{\omega}^{n-1} 1_{U_{j}} ; 1_{B_{L / 2}(x)}\right)_{m} .
\end{aligned}
$$

By Theorem 1.8 of [30] on p. 290, there exists a constant $C>0$ such that for all $\omega \in \Omega$ and any open sets $U, B \subset \mathbb{R}^{d}$ :

$$
\left(P_{\omega}^{n-1} 1_{U} ; 1_{B}(x)\right)_{m} \leqslant \sqrt{m(B)} \sqrt{m(U)} \exp \left\{-\frac{\rho(B, U)^{2}}{4 C(n-1)}\right\}
$$

where $\rho(\cdot, \cdot)$ is a pseudo metric on $\mathbb{R}^{d}$, which is defined for open subset $F, F^{\prime} \subset$ $\mathbb{R}^{d}$ through $\rho\left(F, F^{\prime}\right)=\sup \left\{\psi\left(F, F^{\prime}\right): \quad \psi \in C_{c}^{\infty}\left(\mathbb{R}^{d}, \mathbb{R}\right), d \Gamma(\psi, \psi)<d m\right\}$, with $\psi\left(F, F^{\prime}\right) \stackrel{\text { def }}{=} \inf \left\{|\psi(x)-\psi(y)|: x \in F, \quad y \in F^{\prime}\right\}$, cf. p. 290 in [30], and see p. 277 in [30] for the definition of $\Gamma(\cdot, \cdot)$. For our $\mathscr{E}_{m}$, one can easily compute that $d \Gamma(\psi, \psi)=$ $\mathrm{e}^{2 \lambda \ell \cdot x}|\nabla \psi|^{2} d x$ for $\psi \in C_{c}^{\infty}\left(\mathbb{R}^{d}, \mathbb{R}\right)$. Thereafter, we obtain that $\rho\left(F, F^{\prime}\right) \geqslant \inf \{\mid x-$ $y \mid: x \in F, y \in F^{\prime}$ \}. (See also the second example on p. 278 in [30].) Actually, the Dirichlet form $\mathscr{E}_{m}$ plays the role of $\mathscr{E}$, and $\mathscr{E}_{m_{\omega}}$ the role of $\mathscr{E}_{t}$ in [30]. They are symmetric and strongly local, hence with (4.4) the condition (UP) on p. 279, and the assumption for $\mathscr{E}$ on p. 277 in [30] are fulfilled.

Through simple computation, we get that for all $x \in \mathbb{R}^{d}$ and $\omega \in \Omega$

$$
\begin{aligned}
& m\left(B_{L / 2}(x)\right) \leqslant c \mathrm{e}^{2 \lambda \ell \cdot x} \mathrm{e}^{\lambda L} L^{d}, \\
& m\left(U_{0}(L)\right) \leqslant \tilde{c} \mathrm{e}^{2 \lambda \ell \cdot x} \mathrm{e}^{5 \lambda L} L^{2 d-2}, \quad m\left(U_{-}(L)\right) \leqslant \tilde{c} \mathrm{e}^{2 \lambda \ell \cdot x} \mathrm{e}^{-3 \lambda L} L^{2 d-1} .
\end{aligned}
$$

Hence, for all $x \in \mathbb{R}^{d}$ and $\omega \in \Omega$ we obtain from (4.13) that

$$
\mathrm{P}_{x}^{\omega}\left[X_{1} \in B_{L / 2}(x), X_{n} \in U_{-}\right] \leqslant \hat{c} L^{k(d)} \exp \left\{-\frac{\gamma L^{2}}{16 C L}\right\} \leqslant \tilde{c} \mathrm{e}^{-c L},
$$

because $\rho\left(B_{L / 2}(x), U_{-}(L)\right) \geqslant L$ and $n<4 L / \gamma$. Similarly, since $\rho\left(B_{L / 2}(x), U_{0}(L)\right) \geqslant$ $4 L^{2}-L$, we obtain for all $x \in \mathbb{R}^{d}$ and $\omega \in \Omega$ that

$$
\mathrm{P}_{x}^{\omega}\left[X_{1} \in B_{L / 2}(x), X_{n} \in U_{0}\right] \leqslant \hat{c} L^{\tilde{n}(d)} \mathrm{e}^{4 \lambda L} \exp \left\{-c L^{3}\right\} \leqslant \tilde{c} \mathrm{e}^{-c L} .
$$

Collecting the above results, we see that (4.11) is proved.

With the help of the previous two propositions, we obtain:

COROLLARY 4.4. - There exist two constants $c_{3}>0$ and $\tilde{c}_{3}>0$ such that for $m \in \mathbb{N}$,

$$
\sup _{x, \omega} \mathrm{P}_{x}^{\omega}\left[\widetilde{T}_{-2^{m} R}<T_{2^{m} R}\right] \leqslant \tilde{c}_{3} \exp \left\{-c_{3} 2^{m} R\right\}
$$

where $R>0$ is the constant from $R$-separation above (1.6). 
Proof. - Let $4 L=2^{m+1} R$ in the definition of $U(L)$ in (4.8), and observe that $\mathrm{P}_{x}^{\omega}\left[\widetilde{T}_{-2^{m} R}<T_{2^{m} R}\right] \leqslant \mathrm{P}_{x}^{\omega}\left[T_{U} \geqslant 4 L / \gamma\right]+\mathrm{P}_{x}^{\omega}\left[T_{U}<4 L / \gamma, X_{T_{U}} \notin \partial_{+} U\right]$, hence our claim follows immediately from the previous two propositions.

The next two corollaries will be useful when checking the assumptions of Theorems 3.2 and 3.3.

COROLlaRY 4.5. - There exists a constant $c_{4}>0$ such that

$$
\inf _{x, \omega} \mathrm{P}_{x}^{\omega}[D=\infty] \geqslant c_{4}>0
$$

where $D$ is the first backtracking time defined below (2.14).

COROLlaRY 4.6. - The process $\left(X_{t}\right)_{t \geqslant 0}$ is transient and $\mathrm{P}_{x}^{\omega}\left[\lim _{t \rightarrow \infty} \ell \cdot X_{t}=\infty\right]=1$ for all $x \in \mathbb{R}^{d}$ and $\omega \in \Omega$. Hence by Proposition 2.7, $\widehat{\mathrm{P}}_{x}$-a.s. $\tau_{1}<\infty$.

The proof of these two corollaries is just a slight variation on the proof of Corollaries 2.3 and 2.4 in [27], where we apply the Support Theorem of StroockVaradhan, cf. p. 25 in [2], instead of ellipticity directly.

\subsection{Integrability properties}

In this part we use the results from the previous part to prove that $\sup _{x, \omega} \widehat{\mathrm{E}}_{x}^{\omega}\left[\mathrm{e}^{c \tau_{1}}\right]<\infty$ for some $c>0$, and derive the main result of this section. The proof is divided into several propositions.

First, let us introduce the random variable

$$
M \stackrel{\text { def }}{=} \sup \left\{\ell \cdot\left(X_{t}-X_{0}\right): 0 \leqslant t \leqslant \widetilde{T}_{-R}\right\},
$$

i.e. $M$ is the maximal relative displacement of $X$. in the direction $\ell$ before it goes $R$ below its origin. It will turn out that $M$ is an important variable in studying the integrability properties of $\ell \cdot X_{\tau_{1}}$. Because $\inf _{x, \omega} \mathrm{P}_{x}^{\omega}\left[\widetilde{T}_{-R}=\infty\right] \geqslant c_{4}>0$, cf. (4.15), we cannot expect $M<\infty \mathrm{P}_{x}^{\omega}$-a.s. Nevertheless, we have the next proposition.

PROPOSITION 4.7. - There exists a constant $c_{7}>0$ small enough such that

$$
\sup _{x, \omega} \mathrm{E}_{x}^{\omega}\left[\mathrm{e}^{c_{7} M}, \widetilde{T}_{-R}<\infty\right] \leqslant 1-\frac{c_{4}}{2},
$$

where $c_{4}$ is the constant defined in (4.15).

Proof. - With the help of (4.14) the proof of this proposition is a slight variation of the proof of Lemma 4.2 in [27], ( $\widetilde{T}_{-R}$ plays the role of the variable $D$ in (4.5) of [27]).

Now we shall prove the integrability of $\mathrm{e}^{c l \cdot X_{\tau_{1}}}$ under the extended quenched measure $\widehat{\mathrm{P}}_{x}^{\omega}$. We recall the $\left(\mathscr{Z}_{t}\right)_{t \geqslant 0}$-stopping times $\left(V_{k}(a)\right)_{k \geqslant 0},\left(\widetilde{N}_{k}(a)\right)_{k \geqslant 0}$ and $N_{1}(a)$ defined in (2.12), (2.13), and the events $\left(A_{k}\right)_{k \geqslant 0}$ introduced in (2.26).

As we will see in the proof of Theorem 4.9, $\exp \left\{c \ell \cdot\left(X_{N_{1}(a)}-X_{0}\right)-c a\right\}$ will play a key role in studying the integrability of $\exp \left\{c \ell \cdot\left(X_{\tau_{1}}-X_{0}\right)\right\}$ under $\widehat{\mathrm{P}}_{x}^{\omega}$. Let us start with: 
PROPOSITION 4.8. - For each $\tilde{c}_{5}>0$ there is a $c_{5}>0$, such that:

$$
\sup _{\substack{x, \omega \\ a>0}} \widehat{\mathrm{E}}_{x}^{\omega}\left[\exp \left\{c_{5}\left(\ell \cdot\left(X_{N_{1}(a)}-X_{0}\right)-a\right)\right\}\right] \leqslant 1+\tilde{c}_{5} .
$$

Proof. - First, we claim that for each $\tilde{c}_{6}>0$, there exists a $c>0$, which tends to 0 as $\tilde{c}_{6}$ tends to 0 , such that

$$
\sup _{\substack{x, \omega \\ a>0}} \mathrm{E}_{x}^{\omega}\left[\exp \left\{c\left(\ell \cdot\left(X_{\widetilde{N}_{1}(a)}-X_{0}\right)-a\right)\right\}\right] \leqslant 1+\tilde{c}_{6} .
$$

To see this, we observe that because for any $x$ and $\omega, \mathrm{P}_{x}^{\omega}$-a.s. $\lim _{t} \ell \cdot X_{t}=+\infty$, cf. Corollary 4.6, hence $V_{k}(a)<\infty, k \geqslant 0$, we can show with the same proof as the one given in the proof of Proposition 2.7 (instead of $3 R$ we simply use $a$ ) that for all $x, \omega$ and for any $a>0$, P $x_{x}^{\omega}$-a.s. $\widetilde{N}_{1}(a)<\infty$. Notice, (we drop the " $a$ " from all $V_{k}(a)$ and $\widetilde{N}_{1}(a)$ )

$$
\begin{aligned}
& \mathrm{E}_{x}^{\omega}\left[\exp \left\{c \ell \cdot\left(X_{\widetilde{N}_{1}(a)}-X_{0}\right)\right\}\right]=\mathrm{E}_{x}^{\omega}\left[\exp \left\{c \ell \cdot\left(X_{\left\lceil V_{0}\right\rceil}-X_{0}\right)\right\}, \widetilde{N}_{1}=\left\lceil V_{0}\right\rceil\right] \\
& \quad+\sum_{k \geqslant 1} \mathrm{E}_{x}^{\omega}\left[\exp \left\{c \ell \cdot\left(X_{\left\lceil V_{k}\right\rceil}-X_{0}\right)\right\}, \widetilde{N}_{1}=\left\lceil V_{k}\right\rceil\right] .
\end{aligned}
$$

Further, we notice that the first term on the right-hand side is smaller than $\exp \{c(a+$ $R / 2)\}$, since $\ell \cdot\left(X_{V_{0}}-X_{0}\right)=a$ and $\ell \cdot\left(X_{\left\lceil V_{0}\right\rceil}-X_{V_{0}}\right) \leqslant R / 2$ on the event $\left\{\left\lceil V_{0}\right\rceil=\widetilde{N}_{1}\right\}$. We also observe that for $k \geqslant 1, \ell \cdot\left(X_{\left\lceil V_{k}\right\rceil}-X_{V_{k}}\right) \leqslant R / 2$ on the event $\left\{\widetilde{N}_{1}=\left\lceil V_{k}\right\rceil\right\}$; and $\ell \cdot\left(X_{V_{k}}-X_{V_{k-1}}\right) \leqslant R+Z \circ \theta_{V_{k-1}}$, with $Z$ defined in (2.24). So, it follows from the strong Markov property that for $k \geqslant 1$,

$$
\begin{aligned}
& \mathrm{E}_{x}^{\omega}\left[\mathrm{e}^{c \ell \cdot\left(X_{\left\lceil V_{k}\right\rceil}-X_{0}\right)}, \tilde{N}_{1}=\left\lceil V_{k}\right\rceil\right] \leqslant \mathrm{e}^{c R / 2} \mathrm{E}_{x}^{\omega}\left[\exp \left\{c \ell \cdot\left(X_{V_{k}}-X_{0}\right)\right\} ; A_{0}, \ldots, A_{k-1}\right] \\
& \quad \leqslant \mathrm{e}^{c R / 2} \mathrm{E}_{x}^{\omega}\left[\exp \left\{c\left(\ell \cdot\left(X_{V_{k-1}}-X_{0}\right)+Z \circ \theta_{V_{k-1}}+R\right)\right\} ; A_{0}, A_{1}, \ldots, A_{k-1}\right] \\
& \quad \leqslant \mathrm{e}^{c R / 2} \mathrm{E}_{x}^{\omega}\left[\exp \left\{c \ell \cdot\left(X_{V_{k-1}}-X_{0}\right)\right\} ; A_{0}, \ldots, A_{k-2} ; \mathrm{E}_{X_{V_{k-1}}}^{\omega}\left[\mathrm{e}^{c(R+Z)} ; A\right]\right],
\end{aligned}
$$

$\left(A_{0}, \ldots, A_{k-2}\right.$ are omitted when $\left.k=1\right)$. It follows from (A.7) that for $c>0$ small enough $\sup _{x, \omega} \mathrm{E}_{x}^{\omega}\left[\mathrm{e}^{c(Z+R)} ; A\right] \leqslant 1-c_{0} / 4$, where the constant $c_{0}>0$ is defined in (2.25). Therefore, by induction we observe that the last expression is smaller than

$$
\mathrm{e}^{c R / 2}\left(1-\frac{c_{0}}{4}\right)^{k} \mathrm{E}_{x}^{\omega}\left[\exp \left\{c \ell \cdot\left(X_{V_{0}}-X_{0}\right)\right\}\right]=\mathrm{e}^{c(a+R / 2)}\left(1-\frac{c_{0}}{4}\right)^{k} .
$$

Hence, for $c>0$ small enough we obtain that

$$
\sup _{\substack{x, \omega \\ a>0}} \mathrm{E}_{x}^{\omega}\left[\exp \left\{c \ell \cdot\left(X_{\widetilde{N}_{1}(a)}-X_{0}\right)-c a\right\}\right] \leqslant \mathrm{e}^{c R / 2} \sum_{k \geqslant 0}\left(1-\frac{c_{0}}{4}\right)^{k}=: C<\infty .
$$

To get (4.19), we observe that by Chebychev's inequality, for $\hat{c} \in(0, c)$,

$$
\sup _{\substack{x, \omega \\ a}} \mathrm{E}_{x}^{\omega}\left[\exp \left\{\hat{c} \ell \cdot\left(X_{\widetilde{N}_{1}(a)}-X_{0}\right)-\hat{c} a\right\}\right] \leqslant 1+\hat{c} C \int_{0}^{\infty} d z \mathrm{e}^{\hat{c} z} \mathrm{e}^{-c z} \leqslant 1+\tilde{c}_{6},
$$

provided $\hat{c}$ is small enough. This proves (4.19). 
Now, observe that it follows from the definition of $N_{1}(a)$ in (2.13) and the strong Markov property, cf. Corollary 2.2, that

$$
\begin{aligned}
& \widehat{\mathrm{E}}_{x}^{\omega}\left[\exp \left\{c \ell \cdot\left(X_{N_{1}(a)}-X_{0}\right)\right\}\right]=\sum_{k \geqslant 1} \widehat{\mathrm{E}}_{x}^{\omega}\left[\mathrm{e}^{c \ell \cdot\left(X_{N_{k}(a)}-X_{0}\right)} ; N_{1}(a)=\widetilde{N}_{k}(a)\right] \\
& =\widehat{\mathrm{E}}_{x}^{\omega}\left[\exp \left\{c \ell \cdot\left(X_{\widetilde{N}_{1}(a)}-X_{0}\right)\right\} ; \lambda_{\widetilde{N}_{1}(a)}=1\right]+\sum_{k \geqslant 1} \widehat{\mathrm{E}}_{x}^{\omega}\left[\exp \left\{c \ell \cdot\left(X_{\widetilde{N}_{k}}(a)-X_{0}\right)\right\} ;\right. \\
& \left.\left.\lambda_{\widetilde{N}_{1}(a)}=\cdots=\lambda_{\widetilde{N}_{k}(a)}=0 ; \widehat{E}_{X_{\widetilde{N}_{k}(a)}}^{\omega}[0] \exp \left\{c \ell \cdot\left(X_{\widetilde{N}_{1}(3 R)}-X_{0}\right)\right\} ; \lambda_{\widetilde{N}_{1}(3 R)}=1\right]\right] .
\end{aligned}
$$

Using Hölder's inequality, we can find $c_{6}>0$ such that $\widehat{\mathrm{E}}_{x, 0}^{\omega}\left[\exp \left\{c_{6} \ell \cdot\left(X_{\widetilde{N}_{1}(a)}-X_{0}\right)-\right.\right.$ $\left.\left.c_{6} a\right\}\right]<1+\tilde{c}_{6}$. Further, we observe that under the measure $\widehat{\mathrm{P}}_{x, \lambda}^{\omega}$, for any integer-valued $\left(\mathscr{F}_{t}\right)_{t \geqslant 0}$-stopping time $S, \lambda_{S}$ is independent of $\mathscr{F}_{S} \otimes \mathscr{S}_{S-1}$, see property (2) of Theorem 2.1. Therefore, we see that for $c \in\left(0, c_{6}\right)$ the previous expression is smaller than

$$
\begin{aligned}
& \varepsilon \widehat{\mathrm{E}}_{x}^{\omega}\left[\exp \left\{c \ell \cdot\left(X_{\widetilde{N}_{1}(a)}-X_{0}\right)\right\}\right] \\
& \quad+\sum_{k \geqslant 1} \hat{\mathrm{E}}_{x}^{\omega}\left[\exp \left\{c \ell \cdot\left(X_{\widetilde{N}_{k}(a)}-X_{0}\right)\right\} ; \lambda_{\widetilde{N}_{1}(a)}=\cdots=\lambda_{\widetilde{N}_{k}(a)}=0\right] \mathrm{e}^{3 c R}\left(1+\tilde{c}_{6}\right) \varepsilon,
\end{aligned}
$$

where $\varepsilon$ is given in (2.5). By induction we obtain that the last expression is

$$
\leqslant \widehat{\mathrm{E}}_{x}^{\omega}\left[\mathrm{e}^{c \ell \cdot\left(X_{\widetilde{N}_{1}(a)}-X_{0}\right)}\right]\left\{\varepsilon+\frac{\varepsilon}{1-\varepsilon} \sum_{k \geqslant 1}\left[(1-\varepsilon) \mathrm{e}^{3 c R}\left(1+\tilde{c}_{6}\right)\right]^{k}\right\} \leqslant \mathrm{e}^{c a} C<\infty,
$$

for some $C>0$ independent of $a$, provided $\tilde{c}_{6}>0$ and $c>0$ are small enough. That is, $\sup _{x, \omega, a} \widehat{\mathrm{E}}_{x}^{\omega}\left[\exp \left\{c \ell \cdot\left(X_{N_{1}(a)}-X_{0}\right)-c a\right\}\right] \leqslant C<\infty$. Our claim follows by a similar computation as in (4.20).

THEOREM 4.9. - There exists a constant $c_{8}>0$ such that

$$
\sup _{x, \omega} \widehat{\mathrm{E}}_{x}^{\omega}\left[\exp \left\{c_{8} \ell \cdot\left(X_{\tau_{1}}-X_{0}\right)\right\}\right]<\infty .
$$

Proof. - Observe that

$$
\begin{aligned}
& \widehat{\mathrm{E}}_{x}^{\omega}\left[\exp \left\{c \ell \cdot\left(X_{\tau_{1}}-X_{0}\right)\right\}\right]=\sum_{k \geqslant 1} \hat{\mathrm{E}}_{x}^{\omega}\left[\mathrm{e}^{c \ell \cdot\left(X_{S_{k}}-X_{0}\right)}, S_{k}<\infty, D \circ \theta_{S_{k}}=\infty\right] \\
& \leqslant \sum_{k \geqslant 1} \hat{\mathrm{E}}_{x}^{\omega}\left[\mathrm{e}^{c \ell \cdot\left(X_{S_{k}}-X_{0}\right)}, S_{k}<\infty\right] \stackrel{\text { def }}{=} \sum_{k \geqslant 1} h_{k},
\end{aligned}
$$

and because for any $x$ and $\omega, \ell \cdot\left(X_{S_{1}}-X_{N_{1}(3 R)}\right) \leqslant 10 R, \widehat{\mathrm{P}}_{x}^{\omega}$-a.s. (cf. Theorem 2.1), Proposition 4.8 implies that $h_{1}<\infty$. So it suffices to show that $\sum_{k \geqslant 1} h_{k+1}<\infty$. To show this, we observe that (cf. (2.15))

$$
\ell \cdot\left(X_{S_{k+1}}-X_{0}\right) \leqslant 10 R+\ell \cdot\left(X_{R_{k}}-X_{0}\right)+\ell \cdot\left(X_{N_{1}\left(a_{k}\right)}-X_{0}\right) \circ \theta_{R_{k}},
$$

with $a_{k}=M\left(R_{k}\right)-\ell \cdot\left(X_{R_{k}}-X_{0}\right)+R \in \mathscr{Z}_{R_{k}}$, (in fact for any $m \geqslant 1, a_{k} \cdot 1_{\left\{R_{k}=m\right\}}$ is $\mathscr{F}_{m} \otimes \mathscr{S}_{m-1}$-measurable, and $\lambda_{m}$ is independent of $\left.\mathscr{F}_{m} \otimes \mathscr{S}_{m-1}\right)$, see also Fig. 3. We 
recall that the shift $\theta_{R_{k}}$ is not applied to $a_{k}$. Therefore, by the strong Markov property, cf. Corollary 2.2, we have:

$$
\begin{aligned}
& \widehat{\mathrm{E}}_{x}^{\omega}\left[\exp \left\{c \ell \cdot\left(X_{S_{k+1}}-X_{0}\right)\right\}, S_{k+1}<\infty\right] \\
& \quad \leqslant \mathrm{e}^{10 c R} \widehat{\mathrm{E}}_{x}^{\omega}\left[\exp \left\{c \ell \cdot\left(X_{R_{k}}-X_{0}\right)\right\}, R_{k}<\infty ; \widehat{\mathrm{E}}_{X_{R_{k}}}^{\omega}\left[\exp \left\{c \ell \cdot\left(X_{N_{1}\left(a_{k}\right)}-X_{0}\right)\right\}\right]\right] \\
& \quad \leqslant \mathrm{e}^{10 c R} \widehat{\mathrm{E}}_{x}^{\omega}\left[\exp \left\{c \ell \cdot\left(X_{R_{k}}-X_{0}\right)\right\}, R_{k}<\infty ;\left(1+\tilde{c}_{5}\right) \mathrm{e}^{c a_{k}}\right],
\end{aligned}
$$

where we applied Proposition 4.8 in the last step, provided $c \in\left(0, c_{5}\right)$.

From Fig. 3 we also observe that with $M$ from (4.16) and $Z$ from (2.24), the following inequalities hold, when $R_{k}$ is finite:

$$
\begin{aligned}
& a_{k} \leqslant Z \circ \theta_{\tilde{T}_{-R}} \circ \theta_{S_{k}}+M \circ \theta_{S_{k}}+2 R, \\
& \ell \cdot\left(X_{R_{k}}-X_{0}\right)=\ell \cdot\left(X_{S_{k}}-X_{0}\right)+\underbrace{\left(\ell \cdot\left(X_{D}-X_{0}\right)\right)}_{\leqslant Z \circ \theta_{\tilde{T}_{-R}}} \circ \theta_{S_{k}} .
\end{aligned}
$$

Put them into the rightmost side of (4.23), apply the strong Markov property at time $S_{k}$, cf. Corollary 2.2 (we use the same argument as above that for $m \geqslant 1$, $\exp \left\{c \ell \cdot\left(X_{S_{k}}-\right.\right.$ $\left.\left.X_{0}\right)\right\} \cdot 1_{\left\{S_{k}=m\right\}}$ is $\mathscr{F}_{m} \otimes \mathscr{S}_{m-1}$-measurable, and $\lambda_{m}$ is independent of $\left.\mathscr{F}_{m} \otimes \mathscr{S}_{m-1}\right)$, then use the strong Markov property for the process $\left(X_{t}\right)_{t \geqslant 0}$ at time $\widetilde{T}_{-R}$ on the event it is finite, we obtain (observe $M$ is $\mathscr{F}_{\tilde{T}_{-R}}$-measurable)

$$
\begin{aligned}
& \widehat{\mathrm{E}}_{x}^{\omega}\left[\exp \left\{c \ell \cdot\left(X_{S_{k+1}}-X_{0}\right)\right\} ; S_{k+1}<\infty\right] \\
& \quad \leqslant \mathrm{e}^{12 c R} \widehat{\mathrm{E}}_{x}^{\omega}\left[\mathrm{e}^{c \ell \cdot\left(X_{S_{k}}-X_{0}\right)}, S_{k}<\infty,\left(1+\tilde{c}_{5}\right) \widehat{\mathrm{E}}_{X_{S_{k}}}^{\omega}\left[\exp \left\{c\left(2 Z \circ \theta_{\tilde{T}_{-R}}+M\right)\right\}, \widetilde{T}_{-R}<\infty\right]\right] \\
& \quad \leqslant \mathrm{e}^{12 c R} \widehat{\mathrm{E}}_{x}^{\omega}\left[\mathrm{e}^{c \ell \cdot\left(X_{S_{k}}-X_{0}\right)}, S_{k}<\infty,\left(1+\tilde{c}_{5}\right) \widehat{\mathrm{E}}_{X_{S_{k}}}^{\omega}\left[\mathrm{e}^{c M} \mathrm{E}_{X_{\tilde{T}_{-R}}^{\omega}}\left[\mathrm{e}^{2 c Z}\right], \widetilde{T}_{-R}<\infty\right]\right] .
\end{aligned}
$$

From (A.6) we know, for $c>0$ small enough, $\sup _{x, \omega} \widehat{\mathrm{E}}_{x}^{\omega}\left[\mathrm{e}^{2 c Z}\right] \leqslant 1+\tilde{c}_{5}$. Hence it follows from (4.17) that the last expression is

$$
\begin{aligned}
& \leqslant \mathrm{e}^{12 c R} \widehat{\mathrm{E}}_{x}^{\omega}\left[\mathrm{e}^{c \ell \cdot\left(X_{S_{k}}-X_{0}\right)}, S_{k}<\infty,\left(1+\tilde{c}_{5}\right)^{2} \mathrm{E}_{X_{S_{k}}}^{\omega}\left[\mathrm{e}^{c M}, \widetilde{T}_{-R}<\infty\right]\right] \\
& \leqslant \mathrm{e}^{12 c R}\left(1+\tilde{c}_{5}\right)^{2}\left(1-\frac{c_{4}}{2}\right) \widehat{\mathrm{E}}_{x}^{\omega}\left[\mathrm{e}^{c \ell \cdot\left(X_{S_{k}}-X_{0}\right)}, S_{k}<\infty\right] \\
& \leqslant(1-\alpha) \widehat{\mathrm{E}}_{x}^{\omega}\left[\exp \left\{c \ell \cdot\left(X_{S_{k}}-X_{0}\right)\right\}, S_{k}<\infty\right],
\end{aligned}
$$

for some $\alpha>0$, provided $\tilde{c}_{5}>0$ and $c \in\left(0, c_{5}\right)$ are small enough such that $\mathrm{e}^{12 c R}(1+$ $\left.\tilde{c}_{5}\right)^{2}\left(1-c_{4} / 2\right)<1-\alpha$. By induction the last expression is:

$$
\leqslant(1-\alpha)^{k} \hat{\mathrm{E}}_{x}^{\omega}\left[\exp \left\{c \ell \cdot\left(X_{S_{1}}-X_{0}\right)\right\}, S_{1}<\infty\right] .
$$

Coming back to (4.22), we obtain

$$
\sup _{x, \omega} \widehat{\mathrm{E}}_{x}^{\omega}\left[\mathrm{e}^{c \ell \cdot\left(X_{\tau_{1}}-X_{0}\right)}\right] \leqslant \sup _{x, \omega} \widehat{\mathrm{E}}_{x}^{\omega}\left[\mathrm{e}^{c \ell \cdot\left(X_{S_{1}}-X_{0}\right)}, S_{1}<\infty\right] \cdot \sum_{k \geqslant 0}(1-\alpha)^{k}<\infty,
$$

because $\sup _{x, \omega} \hat{\mathrm{E}}_{x}^{\omega}\left[\exp \left\{c \ell \cdot\left(X_{S_{1}}-X_{0}\right)\right\}, \quad S_{1}<\infty\right]<\infty$ (cf. the statement below (4.22)). 
As a corollary, we obtain an exponential estimate on the tail of $\tau_{1}$. Let us point out that such an estimate together with Theorem 4.9 and the renewal structure of Theorem 2.5 can be used to derive large deviation controls, see [32,33].

COROLlARY 4.10. - There exist constants $c_{9}>0$ and $\tilde{c}_{9}>0$ such that for $u \in \mathbb{N}$

$$
\sup _{x, \omega} \widehat{\mathrm{P}}_{x}^{\omega}\left[\tau_{1}>u\right] \leqslant \tilde{c}_{9} \exp \left\{-c_{9} u\right\} .
$$

Proof. - Observe that for $u \geqslant 6 R / \gamma, x \in \mathbb{R}^{d}$ and $\omega \in \Omega$ :

$\widehat{\mathrm{P}}_{x}^{\omega}\left[\tau_{1}>u\right] \leqslant \widehat{\mathrm{P}}_{x}^{\omega}\left[\tau_{1}>u, \ell \cdot\left(X_{\tau_{1}}-X_{0}\right) \leqslant \frac{\gamma}{2} u-3 R\right]+\widehat{\mathrm{P}}_{x}^{\omega}\left[\ell \cdot\left(X_{\tau_{1}}-X_{0}\right)>\frac{\gamma}{2} u-3 R\right]$.

By Chebychev's inequality and Theorem 4.9, the last term on the right-hand side is smaller than $\tilde{c} \mathrm{e}^{-c u}$, for some $\tilde{c}>0$ and $c \in\left(0, c_{8}\right)$. Hence it suffices to study the first term on the right-hand side of the above expression. Let $U$ now be the cylinder defined in (4.8), which is centered in $x$, has height $4 L=\gamma u$ in the direction $\ell$ and radius $4 L^{2}$ in the directions normal to $\ell$. With the observation that $\mathrm{P}_{x}^{\omega}$-a.s. $\sup _{s \leqslant \tau_{1}}\left(X_{s}-X_{\tau_{1}}\right)<3 R$, cf. Fig. 4, we see that for all $x \in \mathbb{R}^{d}$ and $\omega \in \Omega$ :

$$
\begin{aligned}
& \widehat{\mathrm{P}}_{x}^{\omega}\left[\tau_{1}>u, \ell \cdot\left(X_{\tau_{1}}-X_{0}\right) \leqslant \frac{\gamma}{2} u-3 R\right] \leqslant \mathrm{P}_{x}^{\omega}\left[T_{(\gamma / 2) u}>u\right] \\
& \quad \leqslant \mathrm{P}_{x}^{\omega}\left[T_{U}<T_{(\gamma / 2) u}\right]+\mathrm{P}_{x}^{\omega}\left[T_{U}=T_{(\gamma / 2) u}>u\right] \\
& \quad \leqslant \mathrm{P}_{x}^{\omega}\left[T_{U} \geqslant u\right]+\mathrm{P}_{x}^{\omega}\left[T_{U}<u, X_{T_{U}} \notin \partial_{+} U\right]+\mathrm{P}_{x}^{\omega}\left[T_{U}=T_{(\gamma / 2) u}>u\right] .
\end{aligned}
$$

Observe that by Proposition 4.2 the first and the third term in the above expression are smaller than $\tilde{c} \mathrm{e}^{-c u}$ for suitable $\tilde{c}>0$ and $c>0$, and by Proposition 4.3 the second term is also smaller than $\tilde{c} \mathrm{e}^{-c u}$. This finishes our proof.

We come now to the main result of this section, namely a law of large numbers and functional central limit theorem under the annealed measure:

THEOREM 4.11. - Let $\left(X_{t}\right)_{t \geqslant 0}$ be the (unique strong) solution to the stochastic differential equation $d X_{t}=d W_{t}+\nabla V\left(X_{t}, \omega\right) d t$ and $X_{0}=x$, where for each $\omega \in \Omega$, $V(\cdot, \omega) \in C^{1}\left(\mathbb{R}^{d}, \mathbb{R}\right)$ has bounded and Lipschitz-continuous derivatives, and $A \mathrm{e}^{2 \lambda \ell \cdot x} \leqslant$ $V(x, \omega) \leqslant B \mathrm{e}^{2 \lambda \ell \cdot x}$ holds for some $\ell \in S^{d-1}, A, B>0$ and $\lambda>0$. Then

$$
\mathrm{P}_{0} \text {-a.s. } \quad \frac{X_{t}}{t} \stackrel{t \rightarrow \infty}{\longrightarrow} v
$$

with a deterministic $v \in \mathbb{R}^{d}$, which is given in (3.7), and $\ell \cdot v>0$; further the processes $\left(\left(X_{s t}-v s t\right) / \sqrt{s}\right)_{t \geqslant 0}$ converge in law under $\mathrm{P}_{0}$, as $s \rightarrow \infty$, to a non-degenerate $d$-dimensional Brownian motion with covariance matrix $\mathbf{K}$ given in (3.12).

Proof. - It follows from (4.15) and Corollary 4.10 that the condition (3.1) is fulfilled. Our claims follow from Theorem 3.2 and Theorem 3.3. 


\section{Acknowledgement}

I am deeply indebted to my advisor Prof. A.-S. Sznitman, who guided me into this area and patiently answered my questions.

\section{Appendix A}

\section{A.1. Some facts about continuous martingales}

LEMMA A.1. - On some probability space $\left(\Omega, \mathscr{F},\left(\mathscr{F}_{t}\right)_{t \geqslant 0}, \mathrm{P}\right)$, let $\left(Y_{t}\right)_{t \geqslant 0}$ be a continuous martingale satisfying $Y_{0}=0$ and $\langle Y\rangle_{t} \leqslant v t$ for $t \geqslant 0$. Then for $p>1$ there is a constant $c(p, v)>0$ such that

$$
\mathrm{E}\left[\sup _{s \leqslant t}\left|Y_{s}\right|^{p}\right] \leqslant c(p, v) t^{p / 2}
$$

and

$$
\text { P-a.s. } \quad \frac{1}{t} \sup _{s \leqslant t}\left|Y_{s}\right| \stackrel{t \rightarrow \infty}{\longrightarrow} 0 .
$$

Proof. - The Bernstein's inequality, cf. pp. 153-154 in [25], shows that

$$
\mathrm{P}\left[\sup _{s \leqslant t}\left|Y_{s}\right| \geqslant a\right] \leqslant 2 \exp \left\{-\frac{a^{2}}{2 v t}\right\}
$$

hence

$$
\mathrm{E}\left[\sup _{s \leqslant t}|Y|^{p}\right] \leqslant p \int_{0}^{\infty} y^{p-1} \exp \left\{-\frac{y^{2}}{2 v t}\right\} d y=: c(p, v) t^{p / 2} .
$$

For (A.2), it suffices to prove that P-a.s. $\frac{1}{n} \sup _{s \leqslant n}\left|Y_{s}\right| \stackrel{n \rightarrow \infty}{\longrightarrow} 0$. To see this, we observe that from (A.3) it follows that for $a>0$

$$
\sum_{n \geqslant 1} \mathrm{P}\left[\frac{1}{n} \sup _{s \leqslant n}\left|Y_{s}\right| \geqslant a\right] \leqslant 2 \sum_{n \geqslant 1} \exp \left\{-\frac{a^{2} n}{2 v}\right\}<\infty
$$

and the claim follows from Borel-Cantelli's lemma.

From this lemma we easily get the next two corollaries.

COROLlaRY A.2. - Let $\left(X_{t}\right)_{t \geqslant 0}$ be the solution of the stochastic differential equation (1.7), whose coefficients satisfy (1.1), (1.3) and (1.4). Then there exist two constants $c>0$ and $\tilde{c}>0$ depending only on $(d, v, \bar{b})$ such that for all $x \in \mathbb{R}^{d}, \omega \in \Omega$ and $L>0$,

$$
\sup _{x, \omega} \mathrm{P}_{x}^{\omega}\left[\sup _{s \leqslant 2}\left|X_{s}-X_{0}\right| \geqslant L\right] \leqslant \tilde{c} \mathrm{e}^{-c L^{2}}
$$


Proof. - Observe that for all $x \in \mathbb{R}^{d}$ and $\omega \in \Omega \mathrm{P}_{x}^{\omega}$-a.s. $X_{t}-X_{0}=\int_{0}^{t} b\left(X_{s}, \omega\right) d s+$ $Y_{t}(\omega)$, with the $\mathrm{P}_{x}^{\omega}$-local martingale $Y_{t}(\omega):=\int_{0}^{t} \sigma\left(X_{s}, \omega\right) d W_{s}$. Further we observe by our assumption (1.4) that $\left\langle Y^{j}(\omega)\right\rangle_{t} \leqslant v t$ for all $j=1, \ldots, d$ and $\omega \in \Omega$. Therefore with our assumption $|b| \leqslant \bar{b}$, it follows immediately from the Bernstein's inequality (A.3) that

$$
\mathrm{P}_{x}^{\omega}\left[\sup _{s \leqslant 1}\left|X_{s}-X_{0}\right| \geqslant L\right] \leqslant \mathrm{P}_{x}^{\omega}\left[\sup _{s \leqslant 1}\left|Y_{s}(\omega)\right| \geqslant(L-\bar{b})\right] \leqslant \tilde{c} \mathrm{e}^{-c L^{2}} .
$$

COROLlARY A.3. - Let $Z(\omega):=\sup _{s \leqslant 1}\left|X_{s}-X_{0}\right|$, then for all $\alpha>0$ there exists $a$ constant $\delta(\alpha, d, v, \bar{b})>0$ such that

$$
\sup _{x, \omega} \mathrm{E}_{x}^{\omega}\left[\mathrm{e}^{\delta Z}\right] \leqslant 1+\alpha .
$$

Further, let $A \in \mathscr{F}_{1}$ be an event such that $\sup _{x, \omega} \mathrm{P}_{x}^{\omega}[A] \leqslant 1-2 \beta$ for some $\beta>0$, then there exists a constant $\delta(\beta, d, v, \bar{b})>0$ such that

$$
\sup _{x, \omega} \mathrm{E}_{x}^{\omega}\left[\mathrm{e}^{\delta Z} ; A\right] \leqslant 1-\beta
$$

Proof. - Because $Z(\omega) \leqslant \sup _{s \leqslant 1}\left|Y_{s}(\omega)\right|+\bar{b}$, we get for $0<\delta<1$ that

$$
\begin{aligned}
\mathrm{E}_{x}^{\omega}\left[\mathrm{e}^{\delta Z}\right] & \leqslant \mathrm{e}^{\delta \bar{b}} \mathrm{E}_{x}^{\omega}\left[\exp \left\{\delta \sup _{s \leqslant 1}\left|Y_{s}\right|\right\}\right] \\
& =\mathrm{e}^{\delta \bar{b}}(1+\delta \int_{0}^{\infty} d a \mathrm{e}^{\delta a} \underbrace{\mathrm{P}_{x}^{\omega}\left[\sup _{s \leqslant 1}\left|Y_{s}\right| \geqslant a\right]}_{\leqslant 2 d \exp \left\{-a^{2} /(2 d v)\right\}}) \leqslant \mathrm{e}^{\delta \bar{b}}(1+\delta c(\bar{b}, \nu, d)),
\end{aligned}
$$

for some $c(\bar{b}, v, d)>0$ and this proves (A.6). To prove (A.7) we observe by Hölder's inequality that for $p, q>0$ such that $1 / p+1 / q=1$ :

$$
\mathrm{E}_{x}^{\omega}\left[\mathrm{e}^{\delta Z} ; A\right] \leqslant \mathrm{E}_{x}^{\omega}\left[\mathrm{e}^{\delta p Z}\right]^{1 / p} \mathrm{P}_{x}^{\omega}[A]^{1 / q} \leqslant(1+\alpha)^{1 / p}(1-2 \beta)^{1 / q} \leqslant 1-\beta,
$$

by choosing $\delta$ small and $p$ large enough.

\section{A.2. Some results about parabolic PDE}

In this part we will collect some results about parabolic partial differential equations, which we use throughout this article. For detailed treatment we refer to the article by Il'in, Kalashnikov and Oleinik, [11], Section 4.

PROPOSITION A.4. - We consider the linear parabolic equation of second order $\frac{\partial u}{\partial t}=L u$, where

$$
L=\sum_{i, j=1}^{d} a_{i j}(x) \frac{\partial^{2}}{\partial x_{i} \partial x_{j}}+\sum_{j=1}^{d} b_{j}(x) \frac{\partial}{\partial x_{j}},
$$

with the coefficients $a_{i j}$ and $b_{k}$ satisfying for all $x, y \in \mathbb{R}^{d}$ 


$$
\begin{aligned}
& \left|a_{i j}(x)-a_{i j}(y)\right|+\left|b_{k}(x)-b_{k}(y)\right| \leqslant C|x-y|^{\delta}, \\
& \left|a_{i j}(x)\right|+\left|b_{k}(x)\right| \leqslant K, \quad a_{i j}(x)=a_{j i}(x), \\
& \sum_{i, j=1}^{d} a_{i j}(x) \xi_{i} \xi_{j} \geqslant \frac{1}{v} \sum_{j=1}^{d} \xi_{j}^{2}, \quad \xi \in \mathbb{R}^{d},
\end{aligned}
$$

for some $C>0, K>0, v>0$ and $\delta>0$. Then there exists a unique fundamental solution $Z(t, x, y)$ of $\partial u / \partial t=L u$, such that for $t \leqslant 1$

$$
|Z(t, x, y)| \leqslant \frac{M}{t^{d / 2}} \exp \left\{-\frac{\mu|x-y|^{2}}{t}\right\}
$$

for some constants $M(\nu, C, K, d, \delta)>0$ and $\mu(\nu, C, K, d, \delta)>0$.

Further, there exist two constants $a(v, C, K, d, \delta)>0$ and $\widetilde{M}(v, C, K, d, \delta)>0$ such that for $|x-y|^{2}<$ at and $t \in(0,1]$

$$
Z(t, x, y) \geqslant \frac{\widetilde{M}}{t^{d / 2}} .
$$

The claims (A.9) and (A.10) are just the statement (4.16) and (4.75) in [11]. The authors of [11] did not state on which the constants $M, \mu, a$ and $\widetilde{M}$ really depend on, but by working through their computation, cf. pp. 63-82, one can see that these constants only depend on $(v, C, K, d, \delta)$.

As a consequence of the previous proposition we get the next corollary.

COROllary A.5. - Let $U^{x}$ and $B^{x}$ be the open set defined in (2.1). Under the assumption (1.1), (1.3) and (1.4), there exist two constants $\widetilde{M}(v, d, \bar{b}, \bar{\sigma}, K)>0$ and $a(v, d, \bar{b}, \bar{\sigma}, K)>0$ (recall the constants $v, d, \bar{b}, \bar{\sigma}$ and $K$ are defined in Section 1$)$, such that for all $\omega \in \Omega, 1 \geqslant t>0$ and $|x-y|^{2} \leqslant a t$, the transition density $p_{\omega}(t, x, y)$ satisfies

$$
p_{\omega}(t, x, y) \geqslant \frac{\widetilde{M}}{t^{d / 2}},
$$

and there exists a constant $\varepsilon(\nu, d, \bar{b}, \bar{\sigma}, R, K)>0$ such that the sub-transition density $p_{\omega, U^{x}}(1, x, y)($ recall $(2.4))$ satisfies

$$
p_{\omega, U^{x}}(1, x, y) \geqslant \frac{2 \varepsilon}{\left|B_{R}\right|},
$$

for all $y \in B^{x}$.

Proof. - With $a_{i j}=\left(\sigma \sigma^{t}\right)_{i j}$ we see from (1.1), (1.3) and (1.4) that the assumptions of Proposition A.4 are fulfilled. Hence, (A.11) follows immediately from Proposition A.4.

To prove (A.12), first we observe that because of (A.10) there is $t_{0} \in(0,1]$ such that $\sqrt{a t_{0}} \leqslant R / 4$ and for all $t \leqslant t_{0}, \widetilde{M} / t^{d / 2} \geqslant\left(2 M / t_{0}^{d / 2}\right) \exp \left\{-\mu R^{2} /\left(16 t_{0}\right)\right\}$ holds, 
in addition the function $t \mapsto M / t^{d / 2} \exp \left\{-\left(\mu R^{2} / 16 t\right)\right\}$ is monotone increasing on $\left\{t: t \leqslant t_{0}\right\}$. Now let $G=B_{R / 2}(x)$ and $y \in B_{\sqrt{a t_{0}}}(x)$, we observe that on the event $\left\{T_{G}<t \leqslant t_{0}\right\}$, the inequality $p_{\omega}\left(t-T_{G}, X_{T_{G}}, y\right) \leqslant M / t^{d / 2} \exp \left\{-\left(\mu R^{2} / 16 t\right)\right\}$ follows from the monotonicity mentioned above. Hence, by Duhamel's formula, cf. p. 331 in [29]:

$$
p_{\omega, G}(t, x, y)=p_{\omega}(t, x, y)-\mathrm{E}_{x}^{\omega}\left[T_{G}<t, p_{\omega}\left(t-T_{G}, X_{T_{G}}, y\right)\right], \quad x, y \in G,
$$

there is $\tilde{\varepsilon}(\nu, d, \bar{b}, \bar{\sigma}, R, K)>0$ so that $p_{\omega, G}(t, x, y) \geqslant \tilde{\varepsilon}>0$, for $t \leqslant t_{0}$ and $|x-y| \leqslant$ $\sqrt{a t}$. By iteration, it is straightforward to see that $\inf _{\omega, y \in B^{x}} p_{\omega, U^{x}}(1, x, y)>0$.

\section{REFERENCES}

[1] R.J. Adler, The Geometry of Random Fields, Wiley, New York, 1981.

[2] R. Bass, Diffusions and Elliptic Operators, Springer-Verlag, Berlin, 1998.

[3] E. Bolthausen, A.-S. Sznitman, Ten Lectures on Random Media, in: DMV-Lectures, Vol. 32, Birkhäuser, Basel, 2002.

[4] F. Comets, O. Zeitouni, A law of large numbers for random walks in random mixing environments, preprint.

[5] A. De Masi, P.A. Ferrari, S. Goldstein, W.D. Wick, An invariance principle for reversible Markov processes. Applications to random motions in random environments, J. Statist. Phys. 55 (1989) 787-855.

[6] R. Durrett, Stochastic Calculus, CRC Press, Boca Raton, 1996.

[7] S.N. Ethier, T.G. Kurtz, Markov Processes, Wiley, New York, 1986.

[8] A. Friedman, Stochastic Differential Equations and Applications, Vol. 1, Academic Press, San Diego, 1975.

[9] M. Fukushima, Dirichlet Forms and Markov Processes, North-Holland, Amsterdam, 1980.

[10] M. Fukushima, D. Stroock, Reversibility of solutions to martingale problems, in: Probability, Statistical Mechanics, and Number Theory, in: Adv. Math. Suppl. Stud., Vol. 9, Academic Press, San Diego, 1986, pp. 107-123.

[11] A.M. Il'in, A.S. Kalashnikov, O.A. Oleinik, Linear equations of the second order of parabolic type, Russian Math. Surveys 17 (1) (1962) 1-143.

[12] V.V. Jikov, S.M. Kozlov, O.A. Oleinik, Homogenization of Differential Operators and Integral Functionals, Springer-Verlag, Berlin, 1994.

[13] C. Kipnis, S.R.S. Varadhan, A central limit theorem for additive functionals of reversible Markov processes and applications to simple exclusions, Comm. Math. Phys. 104 (1986) $1-19$.

[14] T. Komorowski, G. Krupa, On the existence of invariant measure for Lagrangian velocity in compressible environments, J. Statist. Phys. 106 (3-4) (2002) 635-651.

[15] T. Komorowski, S. Olla, On homogenization of time-dependent random flows, Probab. Theory Related Fields 121 (1) (2001) 98-116.

[16] S.M. Kozlov, The method of averaging and walks in inhomogeneous environments, Russian Math. Surveys 40 (1985) 73-145.

[17] C. Landim, S. Olla, H.T. Yau, Convection-diffusion equation with space-time ergodic random flow, Probab. Theory Related Fields 112 (1998) 203-220.

[18] J.L. Lebowitz, H. Rost, The Einstein relation for the displacement of a test particle in a random environment, Stochastic Process. Appl. 54 (1994) 183-196. 
[19] S.A. Molchanov, Lectures on random media, in: Lecture Notes in Math., Vol. 1581, Springer, Berlin, 1994, pp. 242-411.

[20] K. Oelschläger, Homogenization of a diffusion process in a divergence-free random fields, Ann. Probab. 16 (3) (1988) 1084-1126.

[21] S. Olla, Homogenization of diffusion processes in random fields, École Doctorale, École Polytechnique, Palaiseau, 1994.

[22] H. Osada, Homogenization of diffusion processes with random stationary coefficients, in: Lecture Notes in Math., Vol. 1021, Springer, Berlin, 1983, pp. 507-517.

[23] G. Papanicolaou, S.R.S. Varadhan, Boundary value problems with rapidly oscillating random coefficients, in: J. Fritz, D. Szasz (Eds.), Random Fields, in: Janyos Bolyai Ser., North-Holland, 1981.

[24] M. Reed, B. Simon, Methods of Modern Mathematical Physics, Vol. I: Functional Analysis, Revised and enlarged edition, Academic Press, San Diego, 1980.

[25] D. Revuz, M. Yor, Continuous Martingales and Brownian Motion, 3rd Edition, SpringerVerlag, Berlin, 1999.

[26] W. Rudin, Functional Analysis, McGraw-Hill, New York, 1973.

[27] L. Shen, Asymptotic properties of certain anisotropic walks in random media, Ann. Appl. Probab. 12 (2) (2002) 477-510.

[28] D. Stroock, Probability Theory, An Analytic View, Cambridge University Press, 1993.

[29] D. Stroock, Diffusion semigroups corresponding to uniformly elliptic divergence form operators, in: Lecture Notes in Math., Vol. 1321, Springer-Verlag, Berlin, 1988, pp. 316347.

[30] K.T. Sturm, Analysis on local Dirichlet Spaces - II. Upper Gaussian estimates for the fundamental solutions of parabolic equations, Osaka J. Math. 32 (1995) 275-312.

[31] A.-S. Sznitman, Brownian Motion, Obstacles and Random Media, Springer-Verlag, Berlin, 1998.

[32] A.-S. Sznitman, Slowdown estimates and central limit theorem for random walks in random environment, J. European Math. Soc. 2 (2000) 93-143.

[33] A.-S. Sznitman, On a class of transient random walks in random environment, Ann. Probab. 29 (2) (2001) 723-764.

[34] A.-S. Sznitman, An effective criterion for ballistic behavior of random walks in random environment, Probab. Theory Related Fields 122 (4) (2002) 509-544.

[35] A.-S. Sznitman, M.P.W. Zerner, A law of large numbers for random walks in random environment, Ann. Probab. 27 (4) (1999) 1851-1869.

[36] O. Zeitouni, Lecture notes on random walks in random environment, St. Flour lecture notes, http://www.ee.technion.ac.il/ zeitouni/ps/notes1.ps, 2001. 